\title{
Digital Divide and E-Inclusion as Challenges of the Information Society - Research Review
}

\author{
Robertina Zdjelar \\ PhD student on Faculty of Organization and Informatics \\ University of Zagreb, Varaždin, Croatia
}

\author{
Nikolina Žajdela Hrustek \\ Faculty of Organization and Informatics \\ University of Zagreb, Varaždin, Croatia
}

rzdjelar@foi.hr

nikolina.zajdela@foi.hr

\begin{abstract}
The issue of e-Inclusion has become an important topic and increasingly gains significance if one takes into account the global trend of digital transformation in the development of the information society and that the digital divide is still present. The reason more for taking the e-Inclusion of all social groups as a challenge has recently been the Covid-19 pandemic and natural disasters (earthquakes, floods) that change the norms of social behavior, require adaptation and create new rules. Users' ability to use digital services and goods as well as open data provided by public and private organizations which ultimately results in greater access to relevant information as well as improved quality of life and are a key factor for the effectiveness of digital transformation and the economic justification of investing in advanced digital technologies, goods and services. The digital divide manifests itself on certain social groups, which is a social problem. However, despite these initiatives, there are still limitations that prevent certain population groups from becoming e-inclusive members of the information society. This paper presents an overview of the analysis results of the research perspective in which scientific and professional papers consider the concepts of e-Inclusion and digital divide as a challenge. The multiperspectiveness and multidimensionality of the concept of e-Inclusion indicates its significant role in the development of the information society.
\end{abstract}

Keywords: e-Inclusion, digital divide, digital inequality, information society, open data, empowerment, impact on quality of life

\section{Introduction}

Development of a global digital society is a common vision shared by all countries, especially in today's pandemic situation caused by the COVID-19 virus, as well as the natural disasters like earthquakes and floods. However, it should be noted that the individual's ability to use digital technology and services, which are the products of digital transformation, whether for personal or business purposes, is critical for creation of information society. Throughout history, societies have been divided into 
various social groups or strata that distinguish people's rights and benefits based on a variety of factors such as ethnicity, race, gender, age, and disability. These distinctions are still important in today's digital-based era. Inequality in access to and use of digital technology and the Internet may play a significant role in the emergence of social inequalities.

The "digital divide" is a term used in literature to describe this phenomenon [1], [2], [3], [4], [5], [6], [7]. We may classify digital divide as a special form of social exclusion. Digital divide and social exclusion are complicated concepts, and most scholars in the field do not agree that this divide can be bridged simply by providing computers or connecting people to the Internet. The term "digital divide" was coined in the mid-1990s and was seen as an indicator of inequality in access to ICT and the Internet, as well as an appeal to society to address the problem of inequality [8]. National Telecommunications and Information Administration (NTIA) [9] of the US Department of Commerce in its second study, Falling Through The Net II: New Data on the Digital Divide, published in 1998, mention this concept among the first. Telephone and computer penetration rates of low-income groups, minorities, women, and the elderly and other vulnerable groups in society were examined in the survey [9]. Early studies were first to identify digital divide as a result of observed inequalities in accessing technology in the general population [9], [10] and majority of research was focused on technical aspect of access to a computer and the Internet, but also other technological devices such as mobile phones and digital television have been studied [11], [12].

A variety of academic studies have been conducted at the macro and micro level related to the "digital divide" [7], [3], [13], [14]. At the macro level, the key causes of the digital divide, according to Bindé [12], include a country's income, availability of technology and costs of ICT and the Internet, digital literacy education in school systems, etc. At the micro level, research shows that socio-cultural and economic factors such as gender, age, ethnicity, place of residence, income levels, social support, educational background, and others have an impact on "digital divide" [13], [15], [16], [17], [18]. Afterwards, researchers clarified that it refers not only to differences in access, but also to disparities among Internet users in terms of their ability to benefit from their use of technology [6].

Various scholars have established different forms and levels of digital divide over time. According to Molnar [19] (2003), there are three different forms of digital divide:

The access divide, also known as the early digital divide, refers to the disparity between those with and those without access to ICT and the Internet.

2) The usage gap, also known as the main digital divide, focuses on those who have access but do not use it, as well as the distinction between users and non-users.

3) The quality of usage, also known as the secondary digital divide, focuses on the disparities in participation rates among those who have access to and use the internet, as well as the distinctions between various types of users. Dewan and Riggins [11] further characterized the digital divide into three levels. The first level of the digital divide encompasses both hardware and software use. The second level is the disparity in IT capacity, or the ability to use technology. One of the most important 
aspects of disparity of use, according to their study, is disparities in computer skill levels. The digital outcome divide is the third level of digital divide, which is caused by the second level digital divide as well as other contextual factors. Van Dijk [4] distinguishes four forms of digital divide:

1) Physical access to ICT and the Internet (have/don't have); 2) Motivational access (want/don't want); 3) Skills access (are able/are not able); 4) Usage access (enough/not enough). Given the numerous studies conducted, it is clear that the digital divide is influenced by a population's socioeconomic status and geographic location and many other factors. This is a complex issue, and some scholars are advocating for solutions focused on new paradigms and multi-dimensional approaches that foster digital opportunity, such as e-Inclusion, as means of bringing people into the digital world [20]. Proponents of e-Inclusion claim that by implementing this approach, which allows development of democracy, mutual understanding, training and education of the economically most disadvantaged groups such as low-income people, the disabled and the unemployed, this gap can close completely or be reduced to a minimum [21]. There are several ways to improve e-Inclusion by ensuring infrastructure, providing skill-building courses, and ensuring social support for those who are not e-included. The motivational access appears to be the most difficult for achieving these goals because it is solely dependent on the will of the individuals.

The pandemic of Covid-19 has pushed society to change its practices and habits by being even more e-included and adopt e-service [22], [23], [24], [25], [26], [27].

Since digital divide and e-Inclusion have been research topics of many authors in the last two decades, they still represent a space for research because the aforementioned problems are multidisciplinary and multidimensional.

\section{Background}

The terms digital divide and e-Inclusion are mentioned in numerous papers listed in relevant scientific citation databases (Scopus and Web of Science). The authors have selected those that assure the multidisciplinary point of view about causes of digital divide and effects on raising e-Inclusion rate.

According to the Eurostat Glossary [28]:

- "Digital divide refers to the distinction between those who have Internet access and are able to make use of new services offered on the World Wide Web, and those who are excluded from these services. At a basic level, the participation of citizens and enterprises in the information society depends on access to information and communication technology (ICT), i.e. the presence of electronic devices, such as computers, and Internet connections..." (https://ec.europa.eu/eurostat/statisticsexplained/index.php/Glossary:Digital_divide)

- "E-inclusion refers to the situation where everyone in society can participate in the information society. This requires affordable access to technologies, the accessibility and usability of ICT tools and services, and the ability and skills of all individuals to use these tools." (https://ec.europa.eu/eurostat/statisticsexplained/index.php?title=Glossary:E-Inclusion). 
The European Commission confirmed in 2016 in Digital Competence Framework that about $40 \%$ of EU population do not have enough digital skills, and $22 \%$ do not have it at all. This category includes vulnerable groups such as the elderly population, lower-educated young people, lower-income families and migrants. In addition, around $32 \%$ of the workforce lacks sufficiently developed digital skills. As a response to the problems related to ageing of population, the Active Ageing policy has been developed by UN and supported by other relevant world institutions.

At the start of research process, the author considered numerous researches, projects and programs on e-Inclusion of adults, the elderly population, vulnerable social groups (migrants, disabled people, people at risk of poverty), lifelong education of which [39]-[43], [45] are more significant. Components of micro causes represent an integrated measurement model of digital inequality from [32] and are the common denominator for all these researches.

The articles are focused in general to possible aspects of solving reasons of digital divide to increase the degree of e-Inclusion of vulnerable population groups. Mendonc [29] regarded digital divide as a social issue and suggested to measure the inequality by composite index (access, skills, intensity of use and material status). Public policy is a mechanism that has to be used for solving recognized social issues, as well as solving digital divide by building digital skills as a key to combating poverty as Huesing and Selhofer [30] suggest. The communication infrastructure has become necessary to assure solving digital divide so the public administration and corporations whose activities are ICT are responsible to assure the Internet access and, while solving personal skills issues is aimed at the individual and educational institutions as Epstein et al. [31] presented. Yu et al. [32] emphasized that continuity in usage of digital staff and services leads to the desired effects of permanent eInclusion and collective awareness of importance of the digital economy and society.

The skills are one of the most needed preconditions for being e-included so the United Nations program and policies focused to lifelong learning and human rights to get the same chances are the base for building the personal capacities [33], [34], [35].

Demographic changes across the world mentioned in research conducted by European Central Bank show that $75 \%$ of workforce is 55 to 74 age old [36].

The European Commission confirmed in 2016 in Digital Competence Framework that about $40 \%$ of EU population do not have enough digital skills, and $22 \%$ do not have it at all. This category includes vulnerable groups such as the elderly population, lower-educated young people, lower-income families and migrants. In addition, around $32 \%$ of the workforce lacks sufficiently developed digital skills. As a response to the problems related to ageing of population, the Active Ageing policy has been developed by UN and supported by other relevant world institutions. The authors Walker and Zaidi specified the domains and the index indicators of active ageing. They consist of a few topics: of being able to get the employment, social engagement, independency, healthy and safe life, capacities and environment assured for active ageing [37]. The importance of including the elderly, not only because of economic activities since they can earn incomes, but in general of being e-Included in social activities by specialized clubs for empowerment and building DS was presented by Naumanen and Tukiainen [38]. Fox [39], Padilla-Góngora, López-Liria, et al [40] and 
Hernández-Encuentra, Pousada, Gómez-Zúñiga [41], have researched the lack of motivation for building digital skills and communication about benefits of digital skills as the main reason of being "offline". According the research concluded that new technologies have to be included in everyday life at the time when users feel that they serve for the personal development, and not when they will be used as a substitution of some lost possibilities (mobility, senses, speed, cognitive ability). The similar conclusion has been found by González, Paz Ramírez, Viadel [42]. Active users of tele-services were active during the education process to keep up the mental condition, being socially included and for lifelong learning. During the educational process it is important to avoid barriers that are caused by a lack of technical social support in acquiring digital skills Xie [43]. Additionally, the barriers can be caused by ages, language, physical ability to use ICT. For those adults who decided to take challenge to build their digital skills it is important who is the trainer. Čurin [44] has concluded according the research that young lecturers often do not understand the special needs and advantages of the elderly. The study found that the elderly finds it easier to work with instructors who are generationally closer to them, and who have one level of more developed digital literacy and skills. It is also important what kind of tools are used during the educational process. Public policies are aimed at reducing the overall digital divide through a number of media literacy projects and e-learning projects, but to no avail due to insufficient systematic methodological access L. AbadAlcalá [45]. Silva, Matos and Martinez-Pecino [46] presented the results of a regression analysis which showed that general stability and public policies have an impact on the will to use the Internet, reinforcing the importance of public policies to encourage e-Inclusion of older people.

R.S. Sharma, et al. "Digital Literacy and Knowledge Societies: A Grounded Theory Investigation of Sustainable Development" [47] have dealt with following issues: a) what are the important policies implemented with a view to promoting digital literacy, digital inclusion and participation in the company known as Sharma $\mathrm{Ja}$; b) how digital literacy policy affects sustainable development (education, health and e-public administration) [48] in 2016. The survey was conducted in Finland, Singapore, New Zealand, Hong Kong and Qatar, also taking into account their socioeconomic parameters (population, GDP, annual GDP growth, country area, net income). Following this research, in 2018 Sharma presented a model of digital literacy maturity [48] which has four pillars (governance, infrastructure, education and human capital, and innovation) and the maturity dimension (the lowest level is access, followed by: use, participation and human values). The conclusion of that research is that public policies by promoting workshops to strengthen digital skills are key to developing an information-based economy.

In order to strengthen digital skills in general, but also with focus on the needs of the elderly, strategies have been planned in this decade, projects have been implemented at national level (albeit in a limited area, such as provinces, large cities, etc.), and countries that have implemented such projects/programs should definitely highlight Spain [45], Finland [49], United Kingdom [50], [51], Czech Republic [52], Lithuania [53], [54], Australia [55], Mexico [56], Canada [57], and United States [58]. For the most part, physical infrastructure projects are the subject of most of the 
countries' strategies in the EU. The European Commission has also supported projects to raise the digital skills of the elderly, including a view to raising their competitiveness in the labor market [59], mainly with a view to reducing poverty rates, which is certainly linked to the results of the European Central Bank survey [59]. The economic impact of digital technology was measured by a composite index that, according to R. Evangelista, P. Guerrieri, V. Meliciani [60] includes three sets of aggregated economic variables: a) labor productivity and GDP growth per capita, b) employment growth, c) employment rates of special groups (women, the elderly and long unemployed), which are rising. During the research, it was concluded that there is an impact of several transmission mechanisms from ICT approach, use and digital empowerment on macroeconomic variables (labor productivity, GDP/population, employability growth and employability rates).

Digital literacy not only represents the ability to use programs or digital devices, but includes cognitive abilities, driving abilities, sociological and emotional skills that users need to be able to operate in a digital environment, so in the Digital Era it is recognized as a survival skill [61].

In 2014, the Commission conducted research concerning the inclusion of the elderly population in society through volunteer work in five EU countries (Denmark, Germany, Italy, the Netherlands and Portugal) [62]. The research focused on volunteering as a tool to prevent social exclusion of the elderly population. The authors cited recommendations for public policy makers in the domain of social policy, but also for municipalities and cities, based on knowledge gathered by the research.

On the other hand, just as Siren and Grønborg Knudsen conclude in their research [63], the key problem of the implementation of e-public administration and e-health projects is precisely the inability of users to use such services.

Although it has been almost twenty years since the problem of the digital divide became a topic for discussion and research, it should be said that the problem remains unresolved in the middle of this decade. Namely, technological progress is made at a higher rate than society is ready to monitor and the gap widens, especially in the area of the use of governmental and non-governmental electronic services as well as the increasing opportunities offered related to the use of open data. The European Commission has adopted the Digital Agenda 2020 and the Digital Single Market Strategy, the implementation of which is annually reported by the European Commission via official websites and written reports. The European Parliamentary Research Service said in December 2015 report [64] that digital (il)literacy is a characteristic of four socio-economic categories: "low-educated", "unemployed", "retired" and "aged 55-74". In 2014, according to Eurostat data, about 50\% of the population aged 55-74 use the Internet. Since open issues persist, the authors decided to make a cross-section of the area by identifying the status according to referenced models, on the one hand digital inequality and on the other e-Inclusion. 


\section{Methodology}

The goal of this research is to present the crosscut of the causes of the digital divide and effects of e-Inclusion by using two groups of key words, first specified by authors of the paper and second by content search made by authors of this paper.

The research questions we ask are:

1) What are the observable themes in published studies on digital divide and eInclusion?

2) What are the observable trends in published studies on digital divide and eInclusion?

Based on the research goal and defined research questions, the research procedure is divided into several steps.

In the first step authors have determined what kind of documents will include in the research. The documents that have been considered in this research are articles, survey reports, project reports, and books which are divided in two groups: scientific and professional papers. For documents questionable regarding classification, the authors were contacted to provide additional information about the classification of the paper.

In the second step, the research concept was defined. This step considered multidisciplinary approach by using components and attributes of multidimensional and multiperspective theoretical model of e-Inclusion made by Žajdela Hrustek [65] and integrated model of digital inequality [32] (in following text: referenced models). The theoretical model of e-Inclusion that was taken as theoretical foundation consists of four components: access, use, empowerment and the impact on quality of life. Another side is theoretical model of digital inequalities that consists of: a) deterrents of digital inequalities divided into macro cause, meso cause and micro cause, and b) measurements of digital inequality: behavioral measurement and effect measurement. This research connects the aforementioned two models by observing the cause-andeffect role of detected key words to e-Inclusion and to digital divide and is presented as theoretical relations.

The third step was related to the decision on which sources will be used for searching papers that have to be analyzed. Authors have used library databases from network sources to find scientific papers, regarding multidisciplinary approach, using keywords such as: accessibility, active ageing, ageing society policy (social policy), competence, digital literacy (ICT literacy, computer literacy), elderly citizens (older people, elders, older age), ICT including mobile telephony and all-inclusive infrastructure, e-Inclusion (digital inclusion, info inclusion), rural areas, learning strategy, later life learning (lifelong learning, lifelong education, adult learning), vulnerable groups (people with disabilities, marginalized groups), empowerment, social support, public community policy, e-readiness, digital divide (digital exclusion), user experience ((UX), individual experience). These keywords are related to inclusion issues and mostly present the cause of digital inequalities. Additionally, authors have checked the references of already identified papers. The next condition is that article had to be published in scientific journals, indexed in Scopus, Web of 
Science (WoS) or other referred sources specified on network sources on Faculty's library webpage.

Authors have researched the respectable sources of relevant institutions official webpages to find professional papers indexed in other sources (official web pages of institutions, official project web pages, etc.).

The point of including both kinds of papers, scientific and professional, is to get the view of which issues are emphasized by scientific research and which by professional.

In the fourth step authors specify keywords that were used for search of databases. There have been specified two groups of key words: a) specified by authors of the paper and b) by content search made by authors of this paper. The documents that do not specify keywords by authors (older scientific articles, professional articles, reports) were excluded from that part of research. For both groups of classification have been created tuple for further comparation.

Other sources indexed databases are: Index Copernicus International and EBSCOhost, Cabell Publishing, Inc., CrossRef, EBSCO, Google Scholar. Books are published by Springer. Conferences: IFIP Advances in Information and Communication Technology, IFIP International Federation for Information Processing, ESA Research Network Sociology of Culture Midterm Conference: Culture and the Making of Worlds, European, Mediterranean \& Middle Eastern Conference on Information Systems.

The key words that researchers and authors of this paper have emphasized are: ageing society (policy), active ageing, elderly citizens/older people, other vulnerable groups, digital literacy, competences/e-readiness, inclusion/e-Inclusion, digital divide, ICT, mobile telephony, learning/informal education/empowerment.

The fifth step involved determining the analysis criteria, and they are grouped in three groups. The first group of research criteria focus on year of publishing, specifying source of the document and indexed database, key words - defined by authors of document, key words - content of the document, county of research, clusters of countries by number of matching in analyzed research.

Second group of research criteria is focused on topics and issues of investigation, data collection method/technique, paper by research population groups. The list of data collection method/techniques is created according to findings. In some researches have been used the mixed method/technique, so the authors have decided to present founded combination. The population groups are presented as they have been found during the research in analyzed documents. There are overlaps between the groups authors decided at the end to few groups: under 20 years, between 25 and 65, over 50 years old, whole population; in some cases the population was not mentioned and in some cases it was not applicable.

The third group of research criteria is defined by multidisciplinary approach for solving problem of digital divide and raising e-Inclusion rate. As the reference models describe, papers are grouped by components and attributes in chronologic array from 2003 to 2020. As it has been mentioned, the papers are divided into two groups: scientific and professional, and are compared with the related components of two reference models (digital inequality and e-Inclusion). 
The collected data were analyzed using descriptive statistical analysis and presented through the tables in the following chapters.

\section{Results}

Summarization and categorization of reviewed papers are grouped by research focus, research approach and by theoretical foundation on referenced models (e-Inclusion model and digital inequality model).

\subsection{Research focus}

Using described criteria, 58 documents have been included in the research, spanning the years 2003 through 2020 (Table 1). Regarding the data shown in Table 1, 51,8\% of papers were published in the last five years. It is interesting to note that after 2014 the number of scientific papers by year is rising. According to the categorization into professional and scientific document, it can be noticed that scientific papers predominate $(86.2 \%)$.

\begin{tabular}{|c|c|c|c|c|}
\hline \multirow{2}{*}{ Year } & \multirow{2}{*}{ Total } & \multirow{2}{*}{$\%$} & \multicolumn{2}{|c|}{ Category of the document } \\
\cline { 4 - 5 } & & & Professional & Scientific \\
\hline 2003 & 1 & 1,72 & & 1 \\
\hline 2007 & 2 & 3,45 & & 2 \\
\hline 2008 & 3 & 5,17 & 1 & 2 \\
\hline 2009 & 1 & 1,72 & & 1 \\
\hline 2010 & 5 & 8,62 & 2 & 3 \\
\hline 2011 & 2 & 3,45 & & 2 \\
\hline 2012 & 2 & 3,45 & & 2 \\
\hline 2013 & 3 & 5,17 & & 3 \\
\hline 2014 & 7 & 12,07 & 1 & 6 \\
\hline 2015 & 2 & 3,45 & & 2 \\
\hline 2016 & 4 & 6,91 & & 4 \\
\hline 2017 & 6 & 10,34 & 1 & 5 \\
\hline 2018 & 6 & 10,34 & 2 & 4 \\
\hline 2019 & 7 & 12,07 & & 7 \\
\hline 2020 & 7 & 12,07 & 1 & 6 \\
\hline Total & 58 & 100 & 8 & 50 \\
\hline
\end{tabular}

Table 1. Papers by year and category of the document

\begin{tabular}{|l|c|c|c|c|c|}
\hline \multicolumn{1}{|c|}{ Journal, Document } & Scopus & WoS & Other & Total & $\%$ \\
\hline Ageing and society & & 1 & & 1 & 1,724 \\
\hline BMC public health & & 1 & & 1 & 1,724 \\
\hline Book & & & 3 & 3 & 5,172 \\
\hline Computer & & 1 & & 1 & 1,724 \\
\hline Comunicar Media Education Research Journal & & & 1 & 1 & 1,724 \\
\hline Conference paper & 3 & & 1 & 4 & 6,897 \\
\hline
\end{tabular}




\begin{tabular}{|c|c|c|c|c|c|}
\hline Educational Gerontology & & 1 & & 1 & 1,724 \\
\hline Employee Relations & & 1 & & 1 & 1,724 \\
\hline European Journal of Ageing & & 1 & & 1 & 1,724 \\
\hline Government Information Quarterly & & 2 & & 2 & 3,448 \\
\hline IEEE Consumer Electronics Magazine & & 1 & & 1 & 1,724 \\
\hline Information Systems Frontiers & & 1 & & 1 & 1,724 \\
\hline Information Systems Management & & 1 & & 1 & 1,724 \\
\hline $\begin{array}{l}\text { International Journal of Business and Social } \\
\text { Science }\end{array}$ & & & 1 & 1 & 1,724 \\
\hline $\begin{array}{l}\text { International Journal of Computer Science and } \\
\text { Information Security }\end{array}$ & & 1 & & 1 & 1,724 \\
\hline International Journal of Electronic Governance & 1 & & & 1 & 1,724 \\
\hline IOSR Journal of Humanities And Social Science & & & 1 & 1 & 1,724 \\
\hline ISG International Society for Gerontechnology & & 1 & & 1 & 1,724 \\
\hline Journal of Aging \& Social Policy & & 1 & & 1 & 1,724 \\
\hline Journal of Medical Internet Research & & 1 & & 1 & 1,724 \\
\hline Lecture Notes in Computer Science & & 6 & & 6 & 10,351 \\
\hline New media \& society & & 1 & & 1 & 1,724 \\
\hline New Zealand Computer Society & & & 1 & 1 & 1,724 \\
\hline PLoS ONE & 1 & & & 1 & 1,724 \\
\hline Procedia-Social and Behavioral Sciences & & & 2 & 2 & 3,448 \\
\hline Profesional de la Información & & 1 & & 1 & 1,724 \\
\hline Project report & & & 3 & 3 & 5,172 \\
\hline Research publication & & & 3 & 3 & 5,172 \\
\hline Revista Latina de Comunicación Social & & 1 & & 1 & 1,724 \\
\hline Rural Society & & 1 & & 1 & 1,724 \\
\hline Science and Engineering Ethics & & & 1 & 1 & 1,724 \\
\hline Sensors & & 1 & & 1 & 1,724 \\
\hline Smart Learning Environments & 1 & & & 1 & 1,724 \\
\hline Statistika-Statistics and Economy Journal & & 1 & & 1 & 1,724 \\
\hline Telecommunications Policy & & 2 & & 2 & 3,448 \\
\hline The Information Society & & 1 & & 1 & 1,724 \\
\hline Topics in Geriatric Rehabilitation & & 1 & & 1 & 1,724 \\
\hline Universal access in the information society & & 1 & & 1 & 1,724 \\
\hline Viešoji Politika Ir adMINISTRAVIMS & & 1 & & 1 & 1,724 \\
\hline ZDM Mathematics Education & & 1 & & 1 & 1,724 \\
\hline Zeitschrift für Weiterbildungsforschung & & & 1 & 1 & 1,724 \\
\hline Total & \multicolumn{2}{|l|}{40} & 18 & 58 & 100 \\
\hline
\end{tabular}

Table 2. Papers by source and citation database.

Table 2 shows the selected papers by source (journals) and the citation database that they are indexed in. The $69 \%$ of papers are published in journals that are indexed in Scopus or WoS. 30\% of other sources are books and project reports. By examination of the list of sources (journals and other types of documents), the previously mentioned can be confirmed - the concepts of digital divide and e-Inclusion are 
multidisciplinary (social science, computer science, information science, medical science and geriatric, etc.), and issues related to these concepts are multidimensional.

The analyzed set of documents are mostly related with concept e-Inclusion, the spatial social group of citizens - elderly, ICT. Ageing and specific vulnerable citizen groups, as well as informal education similarly participate in the sample of analyzed documents.

\begin{tabular}{|l|c|c|}
\hline $\begin{array}{c}\text { Key words - defined by authors of } \\
\text { document }\end{array}$ & Count & $\begin{array}{c}\text { \% of documents } \\
\text { include the key } \\
\text { word }\end{array}$ \\
\hline Inclusion/e-Inclusion/ & 19 & 32,76 \\
\hline Elderly citizens/Older people & 18 & 31,03 \\
\hline ICT & 16 & 27,59 \\
\hline Digital divide & 12 & 20,69 \\
\hline Digital literacy & 7 & 12,07 \\
\hline Ageing society (policy) & 6 & 10,34 \\
\hline Other vulnerable groups & 6 & 10,34 \\
\hline $\begin{array}{l}\text { Learning/Informal } \\
\text { education/Empowerment }\end{array}$ & 6 & 10,34 \\
\hline Active ageing & 4 & 6,9 \\
\hline Competences / E-Readiness & 4 & 6,9 \\
\hline Mobile telephony & 2 & 3,45 \\
\hline
\end{tabular}

Table 3. Key words - defined by authors of documents.

\begin{tabular}{|l|c|c|c|c|c|}
\hline Key words in content of the document & $\begin{array}{c}\text { Scientifi } \\
\mathrm{c}\end{array}$ & $\begin{array}{c}\text { Profes } \\
\text { sional }\end{array}$ & $\begin{array}{c}\text { Tota } \\
\text { ( }\end{array}$ & $\begin{array}{c}\% \text { of all } \\
\text { document } \\
\text { s }\end{array}$ & Rank \\
\hline $\begin{array}{l}\text { Elderly citizens/ Older people/ Elders/ } \\
\text { Older age }\end{array}$ & 42 & 8 & 50 & 86,2 & 1 \\
\hline $\begin{array}{l}\text { e-Inclusion / eInclusion / Digital } \\
\text { inclusion / Info-inclusion }\end{array}$ & 29 & 6 & 35 & 60,3 & 2 \\
\hline $\begin{array}{l}\text { Vulnerable groups / People with } \\
\text { disabilities / Marginalized groups }\end{array}$ & 28 & 7 & 35 & 60,3 & 2 \\
\hline Digital divide Digital exclusion & 28 & 5 & 33 & 56,9 & 4 \\
\hline $\begin{array}{l}\text { Digital literacy / ICT literacy / } \\
\text { Computer literacy }\end{array}$ & 29 & 3 & 32 & 55,2 & 5 \\
\hline $\begin{array}{l}\text { User experience (UX) / Individual } \\
\text { experience }\end{array}$ & 27 & 5 & 32 & 55,2 & 5 \\
\hline $\begin{array}{l}\text { ICT including mobile telephony and all- } \\
\text { inclusive infrastructure }\end{array}$ & 28 & 2 & 30 & 51,7 & 7 \\
\hline Accessibility & 24 & 5 & 29 & 50,0 & 8 \\
\hline Public / Community policy & 22 & 7 & 29 & 50,0 & 8 \\
\hline Competences & 24 & 4 & 28 & 48,3 & 10 \\
\hline Empowerment (Social support) & 7 & 28 & 48,3 & 10 \\
\hline $\begin{array}{l}\text { Later life learning / Lifelong learning / } \\
\text { Lifelong education / Adult learning }\end{array}$ & 22 & 4 & 26 & 44,8 & 12 \\
\hline
\end{tabular}




\begin{tabular}{|l|c|c|c|c|c|} 
Rural areas & 16 & 3 & 19 & 32,8 & 13 \\
\hline Active ageing & 14 & 4 & 18 & 31,0 & 14 \\
\hline Ageing society policy / Social policy & 12 & 3 & 15 & 25,9 & 15 \\
\hline Learning strategy & 7 & 1 & 8 & 13,8 & 16 \\
\hline E-readiness & 3 & 0 & 3 & 5,2 & 17 \\
\hline
\end{tabular}

Table 4. Key words - content of the document.

As it can be seen in Table 4, the content of analyzed documents give a different perspective on keywords than the keywords highlighted by authors. When the digital divide and e-Inclusion are discussed, the interest of scientific and professional community is focused on the elderly citizens and vulnerable societal groups, as well as user experience and digital literacy. That is the landscape of the multidisciplinary and multidimensional researches.

In Table 5 the counties and geographic areas are presented where the researches were conducted. The data are divided national or international research. The $79 \%$ of research papers is related with some specific geographic area. The rest of papers, like reviews and general theory research is classified in the category "Not exactly defined". In absolute value in the UK has been conducted 6 research during the observed period. It is very indicative if we take into the consideration that the rate of e-Inclusion of vulnerable group population by Eurostat is on the top level. Which leads to the conclusion that these groups in the population are most affected by the problem of exclusion from the information society.

\begin{tabular}{|l|c|c|c|c|c|}
\hline $\begin{array}{c}\text { Geographic area in which } \\
\text { the research was conducted }\end{array}$ & National & International & Total & $\%$ & Rank \\
\hline Not exactly defined & 12 & & 12 & 13,04 & 1 \\
\hline EU & & 6 & 6 & 6,52 & 2 \\
\hline United Kingdom & 6 & & 6 & 6,52 & 2 \\
\hline Italy & 3 & 2 & 5 & 5,43 & 4 \\
\hline Netherland & 1 & 4 & 5 & 5,43 & 4 \\
\hline Spain & 3 & 2 & 5 & 5,43 & 4 \\
\hline Denmark & 2 & 2 & 4 & 4,35 & 7 \\
\hline Finland & 2 & 2 & 4 & 4,35 & 7 \\
\hline Germany & & 3 & 3 & 3,26 & 9 \\
\hline Portugal & 1 & 2 & 3 & 3,26 & 9 \\
\hline Romania & 1 & 2 & 3 & 3,26 & 9 \\
\hline Australia & 2 & & 2 & 2,17 & 12 \\
\hline Belgium & & 2 & 2 & 2,17 & 12 \\
\hline Bulgaria & 1 & 1 & 2 & 2,17 & 12 \\
\hline France & & 2 & 2 & 2,17 & 12 \\
\hline New Zeeland & 1 & 1 & 2 & 2,17 & 12 \\
\hline Norway & & 2 & 2 & 2,17 & 12 \\
\hline Slovenia & 2 & & 2 & 2,17 & 12 \\
\hline Switzerland & 1 & 1 & 2 & 2,17 & 12 \\
\hline United States of America & 1 & 1 & 2 & 2,17 & 12 \\
\hline
\end{tabular}




\begin{tabular}{|l|c|c|c|c|c|}
\hline $\begin{array}{l}\text { China (Heilongjiang } \\
\text { Province) }\end{array}$ & 1 & & 1 & 1,09 & 21 \\
\hline Cyprus & & 1 & 1 & 1,09 & 21 \\
\hline Estonia & & 1 & 1 & 1,09 & 21 \\
\hline Greece & & 1 & 1 & 1,09 & 21 \\
\hline Great Britain & & 1 & 1 & 1,09 & 21 \\
\hline Hong Kong SAR & & 1 & 1 & 1,09 & 21 \\
\hline Hungary & 1 & 1 & 1 & 1,09 & 21 \\
\hline Japan & & 1 & 1 & 1,09 & 21 \\
\hline Kenia & 1 & & 1 & 1,09 & 21 \\
\hline Lithuania & 1 & & 1 & 1,09 & 21 \\
\hline Poland & & 1 & 1 & 1,09 & 21 \\
\hline Qatar & 1 & & 1 & 1,09 & 21 \\
\hline Russia & & 1 & 1 & 1,09 & 21 \\
\hline Singapore & & 1 & 1 & 1,09 & 21 \\
\hline Scotland & & 1 & 1 & 1,09 & 21 \\
\hline Slovakia & & 1 & 1 & 1,09 & 21 \\
\hline Sweden & & 1 & 1 & 1,09 & 21 \\
\hline Zimbabwe & 44 & 48 & 92 & 100 & \\
\hline Total & & & & & \\
\hline
\end{tabular}

Table 5. Papers by country.

The importance of the digital divide and the e-Inclusion issues is seen also according to the fact that the researches have been conducted on the wide area (Table 6), everywhere in the world. Sharing the standards, achieved projects, goals and impacts, good and bad practice is noticed during the analysis.

\begin{tabular}{|c|l|}
\hline Count of paper & \multicolumn{1}{|c|}{ Country } \\
\hline 1 & $\begin{array}{l}\text { Belgium; Bulgaria; China; Cyprus; Estonia; France; Greece; Hong } \\
\text { Kong; Hungary; Japan; Kenia; Lithuania; Norway; Poland; Qatar; } \\
\text { Russia; Scotland; Singapore; Sweden; the Slovak Republic; Zimbabwe; }\end{array}$ \\
\hline 2 & Australia; Germany; New Zealand; Slovenia; Switzerland; USA; \\
\hline 3 & Denmark; Portugal; Romania; \\
\hline 4 & Finland; Netherland \\
\hline 5 & Italy; Spain \\
\hline 6 & EU; UK \\
\hline
\end{tabular}

Table 6. Clusters of countries by number of matching in analyzed researches.

Table 7 presents the list of papers by authors and issues of investigation expressed in terms of referenced models. It is evident from the results that according to the causes (macro, meso, micro), most studies have focused on macro causes with emphasis on ideological and personal force. To a much lesser extent, research was focused on behavioral measurement - e-acceptance and effect measurement - situational eInclusion if one looks at it from aspect of causes of digital divide (left side of the Table 
7). While it is interesting to notice this ultimately results in the fact that effects on eInclusion are achieved mostly on the side of access and use and less in terms of empowerment and impact on quality of life (right side of the Table 7).

Researched paper can be divided in 5 groups by research focus of digital inequality and 4 groups by research of e-Inclusion, as it is presented in Table 7.

\begin{tabular}{|c|c|c|c|c|c|c|c|c|c|}
\hline \multirow{2}{*}{\multicolumn{5}{|c|}{\begin{tabular}{|c|} 
Causes of Digital Divide \\
$\begin{array}{c}\text { E-Inclusion or digital divide: an integrated model of } \\
\text { digital inequality }\end{array}$ \\
\end{tabular}}} & \multirow[b]{3}{*}{ Reference of the document } & \multicolumn{4}{|c|}{ Effects on e-Inclusion } \\
\hline & & & & & & \multicolumn{4}{|c|}{$\begin{array}{l}\text { Proposed General Theoretical Conceptual } \\
\text { Model of e-Inclusion }\end{array}$} \\
\hline $\begin{array}{l}\text { MACRO } \\
\text { CAUSE - } \\
\text { Forces }\end{array}$ & $\begin{array}{l}\text { MESO } \\
\text { CAUSE - } \\
\text { Resources }\end{array}$ & $\begin{array}{l}\text { MICRO } \\
\text { CAUSE } \\
- \text { - Access }\end{array}$ & \begin{tabular}{|c|} 
BEHAV \\
IORAL \\
MEASU \\
REMEN \\
T - E- \\
ACCEP \\
TANCE
\end{tabular} & $\begin{array}{c}\text { EFFECT } \\
\text { MEASUR } \\
\text { EMENT - } \\
\text { SITUATI } \\
\text { ONAL E- } \\
\text { INCLUSI } \\
\text { ON }\end{array}$ & & ACCESS & USAGE & $\begin{array}{c}\text { EMPOWER } \\
\text { MENT }\end{array}$ & IMPACT \\
\hline $\begin{array}{l}\text { Personal } \\
\text { force }\end{array}$ & & & $\begin{array}{l}\text { Continu } \\
\text { ous use }\end{array}$ & & $\begin{array}{l}\text { Selwyn, N., Gorard, S., Furlong, } \\
\text { J., \& Madden, L. (2003). }\end{array}$ & $\begin{array}{c}\text { Material } \\
\text { access, } \\
\text { Affordab } \\
\text { ility; }\end{array}$ & $\begin{array}{l}\text { Intensity; } \\
\text { Social } \\
\text { support }\end{array}$ & & \\
\hline $\begin{array}{l}\text { Personal } \\
\text { force }\end{array}$ & \begin{tabular}{|c|}
$\begin{array}{c}\text { Education } \\
\text { al } \\
\text { resource }\end{array}$ \\
\end{tabular} & & & & $\begin{array}{l}\text { Morris, A., Goodman, J., \& } \\
\text { Brading, H. (2007). }\end{array}$ & $\begin{array}{c}\text { Material } \\
\text { access }\end{array}$ & Skills & & $\begin{array}{c}\text { all } \\
\text { atributes }\end{array}$ \\
\hline $\begin{array}{l}\text { Personal } \\
\text { force }\end{array}$ & & $\begin{array}{l}\text { Cognitiv } \\
\text { e access }\end{array}$ & & & \begin{tabular}{|} 
Boulton-Lewis, G. M., Buys, L., \\
Lovie-Kitchin, J., Barnett, K., \& \\
David, L. N. (2007).
\end{tabular} & & \begin{tabular}{|c|} 
Skills; \\
Motivation \\
; Social \\
support \\
\end{tabular} & & \\
\hline $\begin{array}{c}\text { Community } \\
\text { force }\end{array}$ & & & & & $\begin{array}{l}\text { Petrauskas, R-A, Bilevičienè, } \\
\text { T.,Kiškiené, A. (2008) } \\
\end{array}$ & & $\begin{array}{c}\text { Social } \\
\text { support }\end{array}$ & & \\
\hline \multirow[t]{5}{*}{$\begin{array}{c}\begin{array}{c}\text { Personal } \\
\text { force }\end{array} \\
\end{array}$} & & & & & \begin{tabular}{|c|}
$\begin{array}{c}\text { European Social Survey (ESS). } \\
(2008) .\end{array}$ \\
\end{tabular} & & Attitudes & & \\
\hline & & \begin{tabular}{|c|} 
Material \\
access; \\
Motivati \\
onal \\
access \\
\end{tabular} & & & $\begin{array}{l}\text { Repetto, M. and Trentin, G., } \\
\text { (2008). }\end{array}$ & & $\begin{array}{c}\text { Motivation } \\
\text {; Skills }\end{array}$ & & \\
\hline & & $\begin{array}{c}\text { Motivati } \\
\text { onal } \\
\text { access } \\
\end{array}$ & & & $\begin{array}{l}\text { Siren, A., \& Hakamies- } \\
\text { Blomqvist, L. (2009). }\end{array}$ & & Motivation & & \\
\hline & & & $\begin{array}{c}\text { Initial } \\
\text { adoption }\end{array}$ & & $\begin{array}{l}\text { van Deursen A., van Dijk J. } \\
\text { (2010). }\end{array}$ & & Skills & & \\
\hline & & $\begin{array}{c}\text { Material } \\
\text { access; } \\
\text { Motivati } \\
\text { onal } \\
\text { access } \\
\end{array}$ & & & Leahy D., Dolan D. (2010). & & $\begin{array}{c}\text { Motivation } \\
\text {; Skills }\end{array}$ & & \\
\hline \begin{tabular}{|c|}
$\begin{array}{c}\text { Ideological } \\
\text { force }\end{array}$ \\
\end{tabular} & & & & & Maier-Rabler, U., (2010). & & $\begin{array}{c}\text { Social } \\
\text { support }\end{array}$ & & \\
\hline $\begin{array}{c}\text { Ideological } \\
\text { force }\end{array}$ & & & & & Bunker, B. (2010). & & $\begin{array}{c}\text { Social } \\
\text { support }\end{array}$ & & \\
\hline \multirow[t]{2}{*}{\begin{tabular}{|c|}
$\begin{array}{c}\text { Ideological } \\
\text { force }\end{array}$ \\
\end{tabular}} & & & & & European Commission, (2010). & & $\begin{array}{c}\text { Social } \\
\text { support }\end{array}$ & & \\
\hline & $\begin{array}{c}\text { Education } \\
\text { al } \\
\text { resource; } \\
\text { Material } \\
\text { resource; } \\
\text { Psych } \\
\text { resource } \\
\end{array}$ & & & & $\begin{array}{l}\text { Epstein, D., Nisbet, E. C., \& } \\
\text { Gillespie, T. (2011). }\end{array}$ & $\begin{array}{c}\text { Affordab } \\
\text { ility }\end{array}$ & Skills & & \\
\hline $\begin{array}{c}\text { Ideological } \\
\text { force; } \\
\text { Personal } \\
\text { force } \\
\end{array}$ & \begin{tabular}{c|} 
Material \\
resource; \\
Intelectual \\
resource \\
\end{tabular} & $\begin{array}{l}\text { Cognitiv } \\
\text { e access }\end{array}$ & $\begin{array}{l}\text { Continu } \\
\text { ous use }\end{array}$ & & $\begin{array}{c}\text { Almuwil, A., Weerakkody, V., } \\
\text { \& El-Haddadeh, R. (2011). }\end{array}$ & $\begin{array}{c}\text { Material } \\
\text { access }\end{array}$ & \begin{tabular}{|c|} 
Skills; \\
Attitudes; \\
Intensity
\end{tabular} & $\begin{array}{l}\text { Content } \\
\text { creation }\end{array}$ & \\
\hline
\end{tabular}




\begin{tabular}{|c|c|c|c|c|c|c|c|c|c|}
\hline & & & $\begin{array}{c}\text { Initial } \\
\text { adoption }\end{array}$ & & \begin{tabular}{|c|} 
Vishanth Weerakkody, Yogesh \\
K. Dwivedi, Ramzi El- \\
Haddadeh, Ahlam Almuwil \& \\
Ahmad Ghoneim (2012). \\
\end{tabular} & & & & $\begin{array}{c}\text { E- } \\
\text { governm } \\
\text { ent }\end{array}$ \\
\hline & & & & $\begin{array}{l}\text { Impact } \\
\text { personal } \\
\text { life }\end{array}$ & $\begin{array}{l}\text { Näsi, M., Räsänen, P., \& } \\
\text { Sarpila, O. (2012). }\end{array}$ & & Intensity & & $\begin{array}{c}\text { E- } \\
\text { entertain } \\
\text { ment }\end{array}$ \\
\hline & \begin{tabular}{|c|} 
Education \\
al \\
resource
\end{tabular} & & & & Lima Oliveira, A. et.al. (2013). & & Skills & & \\
\hline $\begin{array}{l}\text { Community } \\
\text { force; } \\
\text { Industrial } \\
\text { force; } \\
\text { Personal } \\
\text { force }\end{array}$ & \begin{tabular}{|c|} 
Material \\
resource; \\
Education \\
al \\
resource; \\
Psych \\
resource \\
\end{tabular} & & & & $\begin{array}{l}\text { Rerup Schlichter, B., } \\
\text { Danylchenko, L. (2014). }\end{array}$ & $\begin{array}{c}\text { Material } \\
\text { access; } \\
\text { Affordab } \\
\text { ility; } \\
\text { Network }\end{array}$ & & & \\
\hline $\begin{array}{c}\begin{array}{c}\text { Public } \\
\text { admin } \\
\text { force; } \\
\text { Community } \\
\text { force }\end{array} \\
\end{array}$ & & & & & $\begin{array}{l}\text { Bannier S., Glott R., Meijs V. } \\
\text { (2013). }\end{array}$ & & $\begin{array}{l}\text { Social } \\
\text { support }\end{array}$ & & \\
\hline \multirow[t]{2}{*}{$\begin{array}{l}\text { Personal } \\
\text { force }\end{array}$} & & $\begin{array}{c}\text { Motivati } \\
\text { onal } \\
\text { access } \\
\end{array}$ & & & Ayako, H., Masaaki, K. (2013). & & Motivation & & \\
\hline & & $\begin{array}{l}\text { Cognitiv } \\
\text { e access }\end{array}$ & & & Loureiro A., Barbas M. (2014). & & Skills & & \\
\hline $\begin{array}{c}\text { Personal } \\
\text { force }\end{array}$ & & & & & Billestrup J., Stage J. (2014). & & & \begin{tabular}{|c|} 
E- \\
participation
\end{tabular} & \\
\hline $\begin{array}{c}\text { Ideological } \\
\text { force }\end{array}$ & & & & & Watkins, I., \& Xie, B. (2014). & & Skills & & \\
\hline \multirow[t]{3}{*}{$\begin{array}{c}\text { Ideological } \\
\text { force }\end{array}$} & & $\begin{array}{c}\text { Motivati } \\
\text { onal } \\
\text { access }\end{array}$ & & & Haasjes, M., (2014). & $\begin{array}{c}\text { Affordab } \\
\text { ility }\end{array}$ & \begin{tabular}{|c|} 
Social \\
support; \\
Attitudes; \\
Motivation
\end{tabular} & & \\
\hline & \begin{tabular}{|c|} 
Education \\
al \\
resource \\
\end{tabular} & & & & Nistor, G. (2014). & & Skills & & \\
\hline & \begin{tabular}{|c|} 
Education \\
al \\
resource \\
\end{tabular} & & & & Alcalá, L. A. (2014). & & Skills & & \\
\hline $\begin{array}{c}\text { Community } \\
\text { force }\end{array}$ & & & & & $\begin{array}{c}\text { Comunello F., Mulargia S., } \\
\text { Belotti F., Fernández-Ardèvol } \\
\text { M. (2015). }\end{array}$ & & Skills & & \\
\hline $\begin{array}{c}\text { Ideological } \\
\text { force }\end{array}$ & & & & & $\begin{array}{c}\text { Chalkia E., Bekiaris E., Madrid } \\
\text { R.I. (2015). }\end{array}$ & $\begin{array}{c}\text { Network; } \\
\text { Point of } \\
\text { access }\end{array}$ & & & \\
\hline $\begin{array}{l}\text { Personal } \\
\text { force }\end{array}$ & & & & & \begin{tabular}{|c|} 
Sergeyeva, O., \& Makarova, L. \\
$(2016)$.
\end{tabular} & & Motivation & & \\
\hline \begin{tabular}{|c|} 
Public \\
admin \\
force; \\
Community \\
force
\end{tabular} & & & & & $\begin{array}{l}\text { Sharma, R., Fantin, A. R., } \\
\text { Prabhu, N., Guan, C., \& } \\
\text { Dattakumar, A. (2016). }\end{array}$ & Network & Skills & & \\
\hline \multirow[t]{3}{*}{$\begin{array}{l}\text { Community } \\
\text { force; } \\
\text { Industrial } \\
\text { force; } \\
\text { Personal } \\
\text { force }\end{array}$} & \begin{tabular}{|c|} 
Material \\
resource; \\
Education \\
al \\
resource; \\
Psych \\
resource \\
\end{tabular} & & & & $\begin{array}{l}\text { Berenguer, A., Goncalves, J., } \\
\text { Hosio, S., Ferreira, D., } \\
\text { Anagnostopoulos, T., \& } \\
\text { Kostakos, V. (2016). }\end{array}$ & $\begin{array}{c}\text { Affordab } \\
\text { ility; } \\
\text { Material } \\
\text { access }\end{array}$ & $\begin{array}{c}\text { Motivation } \\
; \text { Skills }\end{array}$ & & \\
\hline & & \begin{tabular}{|c|} 
Material \\
access; \\
Motivati \\
onal \\
access \\
\end{tabular} & & & Friemel, T. N. (2016). & & $\begin{array}{c}\text { Motivation } \\
\text {; Skills }\end{array}$ & & \\
\hline & & $\begin{array}{c}\text { Motivati } \\
\text { onal } \\
\text { access }\end{array}$ & & & $\begin{array}{l}\text { M Viñarás-Abad, L Abad- } \\
\text { Alcalá, C Llorente-Barroso, M }\end{array}$ & & Motivation & & \\
\hline
\end{tabular}




\begin{tabular}{|c|c|c|c|c|c|c|c|c|c|}
\hline & & & & & $\begin{array}{l}\text { Sánchez-Valle, M Pretel- } \\
\text { Jiménez (2017). }\end{array}$ & & & & \\
\hline & & & & $\begin{array}{l}\text { Impact } \\
\text { personal } \\
\text { life }\end{array}$ & $\begin{array}{l}\text { Dudek, H., \& Szczesny, W. } \\
\text { (2017). }\end{array}$ & & Attitudes & & $\begin{array}{c}\text { All } \\
\text { attributes }\end{array}$ \\
\hline & & $\begin{array}{c}\text { Motivati } \\
\text { onal } \\
\text { access }\end{array}$ & & & $\begin{array}{l}\text { Siren, A., \& Knudsen, S. G. } \\
\text { (2017). }\end{array}$ & & Motivation & & \\
\hline \begin{tabular}{|c|}
$\begin{array}{c}\text { Public } \\
\text { admin } \\
\text { force; } \\
\text { Community } \\
\text { force }\end{array}$ \\
\end{tabular} & & & & & $\begin{array}{c}\text { Silva P, Matos AD, Martinez- } \\
\text { Pecino R (2017). }\end{array}$ & & $\begin{array}{l}\text { Social } \\
\text { support }\end{array}$ & & \\
\hline $\begin{array}{l}\text { Personal } \\
\text { force }\end{array}$ & & & & & $\begin{array}{c}\text { Padilla-Góngora, D., López- } \\
\text { Liria, R., del Pilar Díaz-López, } \\
\text { M., Aguilar-Parra, J. M., } \\
\text { Vargas-Muñoz, M. E., \& } \\
\text { Rocamora-Pérez, P. (2017). }\end{array}$ & & Skills & & \\
\hline $\begin{array}{c}\text { Ideological } \\
\text { force }\end{array}$ & \begin{tabular}{|l|} 
Material \\
resource \\
\end{tabular} & $\begin{array}{c}\text { Material } \\
\text { access }\end{array}$ & $\begin{array}{c}\text { Initial } \\
\text { adoption }\end{array}$ & & Dolničar, V., Setinc, M. (2017). & & $\begin{array}{c}\text { Social } \\
\text { support }\end{array}$ & & \\
\hline $\begin{array}{c}\text { Community } \\
\text { force }\end{array}$ & & & & & $\begin{array}{c}\text { Galdon Clavell G., Zamorano } \\
\text { M.M., Zavala Pérez J.M. } \\
\text { (2018). }\end{array}$ & & $\begin{array}{l}\text { Social } \\
\text { support }\end{array}$ & & \\
\hline $\begin{array}{c}\text { Industrial } \\
\text { force }\end{array}$ & & & & & $\begin{array}{l}\text { Ruiz-Rodríguez, F., Lucendo- } \\
\text { Monedero, A.L., González- } \\
\text { Relaño, R. (2018). }\end{array}$ & & Motivation & $\begin{array}{c}\text { Content } \\
\text { creation; } \\
\text { Networking }\end{array}$ & \\
\hline $\begin{array}{l}\text { Personal } \\
\text { force }\end{array}$ & & & & & $\begin{array}{l}\text { Chipeva, P., Cruz-Jesus, F., } \\
\text { Oliveira, T., \& Irani, Z. (2018). }\end{array}$ & & Attitudes & & \\
\hline & & & $\begin{array}{l}\text { Continu } \\
\text { ous use }\end{array}$ & & $\begin{array}{l}\text { Trilar, J., Kos, A., Jazbinšek, S., } \\
\text { Jensterle, L., \& Stojmenova } \\
\text { Duh, E. (2018). }\end{array}$ & & Intensity & & \\
\hline $\begin{array}{l}\text { Ideological } \\
\text { force; } \\
\text { Personal } \\
\text { force }\end{array}$ & & $\begin{array}{c}\text { Motivati } \\
\text { onal } \\
\text { access }\end{array}$ & & & $\begin{array}{c}\text { Centre for Economics and } \\
\text { Business Research (Cebr) } \\
\text { (2018) }\end{array}$ & & & \begin{tabular}{|c|} 
E- \\
participation \\
; E- \\
democracy; \\
Networking; \\
Content \\
creation \\
\end{tabular} & \\
\hline \multirow[t]{3}{*}{\begin{tabular}{|c|} 
Ideological \\
force; \\
Personal \\
force \\
\end{tabular}} & $\begin{array}{c}\text { Interperso } \\
\text { nal } \\
\text { resource }\end{array}$ & $\begin{array}{c}\text { Motivati } \\
\text { onal } \\
\text { access }\end{array}$ & & & Davidson, S., (2018). & & $\begin{array}{c}\text { Attitudes; } \\
\text { Social } \\
\text { support }\end{array}$ & & \\
\hline & \begin{tabular}{|c|}
$\begin{array}{c}\text { Education } \\
\text { al } \\
\text { resource }\end{array}$ \\
\end{tabular} & & & & $\begin{array}{l}\text { Kirongo, A.C., Huka, G. S., } \\
\text { Bundi, D. G., Muketha G. M., } \\
\text { (2019). }\end{array}$ & & Skills & & \\
\hline & & & \begin{tabular}{c|} 
Initial \\
adoption
\end{tabular} & & Brenna, E. (2019). & $\begin{array}{c}\text { Point of } \\
\text { access }\end{array}$ & Intensity & & \begin{tabular}{|c|} 
all \\
atributes \\
\end{tabular} \\
\hline \begin{tabular}{|c|}
$\begin{array}{c}\text { Public } \\
\text { admin } \\
\text { force; } \\
\text { Community } \\
\text { force }\end{array}$ \\
\end{tabular} & & & & & $\begin{array}{l}\text { Al-Muwil, A., Weerakkody, V., } \\
\text { El-haddadeh, R. et al. (2019). }\end{array}$ & & $\begin{array}{l}\text { Social } \\
\text { support }\end{array}$ & & \\
\hline $\begin{array}{c}\begin{array}{c}\text { Public } \\
\text { admin } \\
\text { force; } \\
\text { Community } \\
\text { force }\end{array} \\
\end{array}$ & & & & & $\begin{array}{l}\text { Atarodi, S., Berardi, A.M., } \\
\text { Toniolo, A-M. (2019). }\end{array}$ & & $\begin{array}{l}\text { Social } \\
\text { support }\end{array}$ & & \\
\hline $\begin{array}{c}\text { Ideological } \\
\text { force }\end{array}$ & $\begin{array}{l}\text { Material } \\
\text { resource }\end{array}$ & & & & $\begin{array}{l}\text { Moreno, L., \& Martinez, P. } \\
\text { (2019). }\end{array}$ & $\begin{array}{c}\text { Accessib } \\
\text { ility }\end{array}$ & \begin{tabular}{|c|}
$\begin{array}{c}\text { Network } \\
\text { and } \\
\text { Quality }\end{array}$ \\
\end{tabular} & & \\
\hline $\begin{array}{c}\text { Ideological } \\
\text { force }\end{array}$ & \begin{tabular}{|c|}
$\begin{array}{c}\text { Education } \\
\text { al } \\
\text { resource }\end{array}$ \\
\end{tabular} & $\begin{array}{l}\text { Cognitiv } \\
\text { e access }\end{array}$ & & & Richards, C. (2019). & & \begin{tabular}{|c|} 
Attitudes; \\
Social \\
support \\
\end{tabular} & & \\
\hline $\begin{array}{l}\text { Ideological } \\
\quad \text { force; } \\
\text { Public } \\
\text { admin force }\end{array}$ & & & & & Arrieta F. (2019). & $\begin{array}{c}\text { Affordab } \\
\text { ility }\end{array}$ & $\begin{array}{l}\text { Social } \\
\text { support }\end{array}$ & & \\
\hline
\end{tabular}




\begin{tabular}{|c|c|c|c|c|c|c|}
\hline & $\begin{array}{c}\text { Education } \\
\text { al } \\
\text { resource }\end{array} \mid$ & & $\begin{array}{l}\text { Zhang, X., Tlili, A., } \\
\text { Nascimbeni, F. et al. (2020). }\end{array}$ & Skills & & \\
\hline \multirow[t]{2}{*}{$\begin{array}{l}\text { Ideological } \\
\text { force }\end{array}$} & $\begin{array}{c}\text { Education } \\
\text { al } \\
\text { resource }\end{array}$ & & $\begin{array}{l}\text { Gal, I., Grotlüschen, A., Tout, } \\
\text { D., Kaiser, G., (2020). }\end{array}$ & Skills & & \\
\hline & & $\begin{array}{l}\text { Impact } \\
\text { personal } \\
\text { life }\end{array}$ & $\begin{array}{c}\text { Bejaković, P., \& Mrnjavac, Ž. } \\
\text { (2020). }\end{array}$ & \begin{tabular}{|c|} 
Digital \\
engageme \\
$\mathrm{nt}$
\end{tabular} & $\begin{array}{c}\text { Content } \\
\text { creation; } \\
\text { Networking }\end{array}$ & E-work \\
\hline $\begin{array}{c}\text { Public } \\
\text { admin } \\
\text { force; } \\
\text { Community } \\
\text { force } \\
\end{array}$ & & & $\begin{array}{l}\text { Guenther, J., Smede, B., \& } \\
\text { Young, M. (2020). }\end{array}$ & $\begin{array}{l}\text { Social } \\
\text { support }\end{array}$ & & \\
\hline $\begin{array}{l}\text { Ideological } \\
\text { force }\end{array}$ & & & $\begin{array}{c}\text { Martínez-Bravo, M. C., Sádaba- } \\
\text { Chalezquer, C., \& Serrano- } \\
\text { Puche, J. (2020). }\end{array}$ & $\begin{array}{l}\text { Social } \\
\text { support }\end{array}$ & & \\
\hline \multirow[t]{2}{*}{$\begin{array}{c}\text { Community } \\
\text { force }\end{array}$} & & & $\begin{array}{c}\text { Sun, X., Yan, W., Zhou, H., } \\
\text { Wang, Z., Zhang, X., Huang, S., } \\
\text { \& Li, L. (2020). }\end{array}$ & $\begin{array}{c}\text { Motivation } \\
\text {, Social } \\
\text { support }\end{array}$ & & \\
\hline & & $\begin{array}{l}\text { Impact } \\
\text { personal } \\
\text { life }\end{array}$ & $\begin{array}{l}\text { Burr, C., Taddeo, M. \& Floridi, } \\
\text { L. (2020) }\end{array}$ & $\begin{array}{c}\text { Social } \\
\text { support; } \\
\text { Motivation } \\
;\end{array}$ & & \\
\hline
\end{tabular}

Table 7. Papers by issue of investigation, referenced in Appendix 1.

According to the data in Table 7 . it could be concluded that $63,79 \%$ of analyzed documents are related with macro cause issues (37 of 58 documents), and it follows by meso and micro cause of digital divide 15 of 58 documents, or 25,86\%). During the research it was reviled that ideological forces was the topic in 11 documents, and it is followed by personal force that was found in 9 documents. Considering the meso cause the educational resources it was found that it has been mentioned in 8 documents. On micro cause motivation has been the topic of research in 7 documents. Considering e-acceptance the initial adoption has been researched in four documents and continuous use in three documents. Impact on personal life, as the effect of measurement situation e-Inclusion property has been analyzed four times.

Regarding the e-Inclusion referent model, it could be concluded that usage has been the research topic in 53 documents (of 58) or 91,38\%, access in 11 documents or $18,97 \%$, empowerment in five $(8,62 \%)$ and impact on quality of life in 4 documents $(6,9 \%)$.

The focus of researchers of access issues was directed to skills (14 documents) and social support (13 documents), while on other e-Inclusion components have been noted lower number of occurrence (access: affordability and material access in two documents; empowerment: content creation and networking in two documents; impact on quality of life all attributes have been found in only once).

\subsection{Research approach}

One of the focuses of the research was to determine the method/methodology or technique of collecting data used by the authors in the research they conducted. Results are presented in the Table 8. At the start it is said that 25 of 58 papers focus on analyses of existing documents (for making reviews; using secondary data), so there are no specific methods or techniques for data collection. The usually used 
methods or techniques for collecting data from individuals are: questionnaire (11 of 58 , including semi-structured), interview ( 9 of 58) followed by survey (4) and focus groups (3).

\begin{tabular}{|l|c|c|}
\hline \multicolumn{1}{|c|}{ Data collecting method/technique } & Count & $\%$ \\
\hline Review & 20 & 34,49 \\
\hline Questionnaire & 8 & 13,80 \\
\hline Interview & 6 & 10,34 \\
\hline Secondary data & 5 & 8,63 \\
\hline Survey & 4 & 6,90 \\
\hline Project & 3 & 5,17 \\
\hline Focus group & 3 & 5,17 \\
\hline Grounded theory method & 2 & 3,46 \\
\hline Experiment & 1 & 1,72 \\
\hline User centred design (UCD) & 1 & 1,72 \\
\hline Secondary data, interview & 1 & 1,72 \\
\hline Grounded theory method, interview, questionnaire & 1 & 1,72 \\
\hline $\begin{array}{l}\text { Experiment, } \\
\text { questionnaire }\end{array}$ & 1 & 1,72 \\
\hline Experiment, questionnaire & 1 & 1,72 \\
\hline Experiment, interview & 1 & 1,72 \\
\hline Total & 58 & 100 \\
\hline
\end{tabular}

Table 8. Papers by data collecting method/technique.

Unit of analysis is not explicitly mentioned because the papers analyze the documents and second source data (22 of 58), which makes about $38 \%$ of all papers. Other 36 papers are based on collected data from individuals of specific age groups, as it stands in Table 9. The data tell the researchers of 11 papers included general population in the research process. Other most occurred population groups included in the research were $60+$ and $65+$. In general, $31 \%$ of papers are focused especially on $50+$ population. The reason is certainly because the population of $50+$ is vulnerable group from the digital inequality point of view, mostly because of lack of required skills (in correlation with Eurostat data).

\begin{tabular}{|c|c|c|c|}
\hline Population groups & Count & & \\
\hline $16+$ & 1 & 1,72 & \multirow{5}{*}{10,34} \\
\hline up to 18 & 1 & 1,72 & \\
\hline $18+$ & 2 & 3,46 & \\
\hline $18-74$ & 1 & 1,72 & \\
\hline $19+$ & 1 & 1,72 & \\
\hline $25-65$ & 1 & 1,72 & 1,72 \\
\hline $50-74$ & 1 & 1,72 & \multirow{5}{*}{31,04} \\
\hline $50+$ & 2 & 3,45 & \\
\hline $55+$ & 1 & 1,72 & \\
\hline $58+$ & 1 & 1,72 & \\
\hline $60+$ & 5 & 8,63 & \\
\hline
\end{tabular}




\begin{tabular}{|c|c|r|r|}
$60-79$ & 1 & 1,72 & \multirow{1}{|}{} \\
\cline { 1 - 3 } $65-81$ & 1 & 1,72 & \\
\hline $65+$ & 6 & 10,35 & \\
\hline General population & 11 & 18,98 & 18,97 \\
\hline Not Applicable & 4 & 6,90 & 6,90 \\
\hline Not specified & 18 & 31,03 & 31,03 \\
\hline Total & 58 & 100 & 100 \\
\hline
\end{tabular}

Table 9. Paper by population groups.

As many other social issues, the heterogeneity of population characteristics (especially age) has an impact on the phenomenon that is being analyzed. If we exclude the researches where the population hasn't been the source of data, the eInclusion issues and digital divide issues have been analyzed mostly as an issue related with population $50+$. This is very indicative, and it could be said that researchers consider the vulnerable population group defined by ages.

\subsection{Theoretical foundation on e-Inclusion model and digital inequality model}

Following part of data presented in Table 10 to Table 14 are the results of documents analyzes according to two referenced models: a) digital inequality and b) e-Inclusion model. The aforementioned present the third part of research - the analysis of multidisciplinary approach for solving problem of digital divide and raise e-Inclusion rate. As the reference models describe, papers are grouped by components and attributes in chronologic array from 2003 to 2020. Following part of research displays selected papers divided into two groups: scientific and professional. The papers were analyzed by key words which were found in the content of the paper and paired with the related components of two reference models (digital inequality and e-Inclusion model).

Theoretical foundation of causes and effects on referenced models is presented in Table 10 that presents the connection of causes of digital inequalities from the left side of keywords in content, and the effects they have on e-Inclusion described by components on the right side of keywords. The yellow-colored cells present the connections between terms from specific points of view. It can be concluded that, except from terms "e-Inclusion" and "digital divide", the term "public/community policy" has been related with all components of e-Inclusion model, but it is related only to macro cause of digital inequality model. Most key words are related with usage in e-Inclusion model, but on the left side of Table 10 it can be seen that all parts of the model by key words are equally represented. In other words, it could be said that most of keywords are related with or are cause of only one component of the digital inequality model. 


\begin{tabular}{|c|c|c|c|c|c|c|c|c|c|}
\hline & $\mathrm{Cau}$ & es of $\mathrm{Di}$ & ital Inequalities & & & & ffects on & e-Inclusio & \\
\hline E-Inclt & sion or digit & $\begin{array}{r}\text { al divide } \\
\text { inec }\end{array}$ & $\begin{array}{l}\text { an integrated } \mathrm{m} \\
\text { uality }\end{array}$ & odel of digital & $\begin{array}{c}\text { Multidisciplinary view } \\
\text { of causes and effect }\end{array}$ & $\begin{array}{r}\text { Prop } \\
\text { Conce }\end{array}$ & $\begin{array}{l}\text { sed Gen } \\
\text { tual Mo }\end{array}$ & $\begin{array}{l}\text { eral Theo } \\
\text { del of e-In }\end{array}$ & $\begin{array}{l}\text { etical } \\
\text { elusion } \\
\end{array}$ \\
\hline $\begin{array}{l}\text { Macro } \\
\text { cause - } \\
\text { Forces }\end{array}$ & $\begin{array}{c}\text { Meso } \\
\text { cause - } \\
\text { Resources }\end{array}$ & $\begin{array}{l}\text { Micro } \\
\text { cause - } \\
\text { Access }\end{array}$ & $\begin{array}{c}\text { Behavioral } \\
\text { measurement } \\
\text { - e-acceptance }\end{array}$ & $\begin{array}{c}\text { Effect } \\
\text { measurement } \\
\text { - Situational } \\
\text { e-Inclusion } \\
\end{array}$ & Keywords & Access & Usage & $\begin{array}{c}\text { Empo- } \\
\text { werment }\end{array}$ & $\begin{array}{l}\text { Impact } \\
\text { on } \\
\text { QoL }\end{array}$ \\
\hline & & & & & Accessibility & & & & \\
\hline & & & & & Active ageing & & & & \\
\hline & & & & & $\begin{array}{c}\begin{array}{c}\text { Ageing society policy / } \\
\text { Social policy }\end{array} \\
\end{array}$ & & & & \\
\hline & & & & & Competences & & & & \\
\hline & & & & & $\begin{array}{c}\text { Digital literacy / } \\
\text { ICT literacy / } \\
\text { Computer literacy }\end{array}$ & & & & \\
\hline & & & & & $\begin{array}{l}\text { Elderly citizens/ } \\
\text { Older people/ } \\
\text { Elders/Older age } \\
\end{array}$ & & & & \\
\hline & & & & & $\begin{array}{c}\text { ICT including mobile } \\
\text { telephony and all- } \\
\text { inclusive } \\
\text { infrastructure } \\
\end{array}$ & & & & \\
\hline & & & & & $\begin{array}{c}\text { e-Inclusion / eInclusion } \\
\text { Digital inclusion/ } \\
\text { Info-inclusion }\end{array}$ & & & & \\
\hline & & & & & Rural areas & & & & \\
\hline & & & & & Learning strategy & & & & \\
\hline & & & & & $\begin{array}{l}\text { Later life learning / } \\
\text { lifelong learning / } \\
\text { lifelong education / } \\
\text { adult learning } \\
\end{array}$ & & & & \\
\hline & & & & & $\begin{array}{l}\text { Vulnerable groups/ } \\
\text { People with } \\
\text { disabilities/ } \\
\text { Marginalized groups } \\
\end{array}$ & & & & \\
\hline & & & & & $\begin{array}{c}\begin{array}{c}\text { Empowerment (Social } \\
\text { support) }\end{array} \\
\end{array}$ & & & & \\
\hline & & & & & $\begin{array}{c}\text { Public / Community } \\
\text { policy }\end{array}$ & & & & \\
\hline & & & & & E-readiness & & & & \\
\hline & & & & & $\begin{array}{c}\text { Digital divide / Digital } \\
\text { exclusion }\end{array}$ & & & & \\
\hline & & & & & $\begin{array}{l}\text { User experience (UX) / } \\
\text { Individual experience }\end{array}$ & & & & \\
\hline
\end{tabular}

Table 10. Multidisciplinary approach for solving problem of digital divide and raise e-Inclusion rate.

Table 11 visualizes the dispersion of papers according to components and attributes of referenced models by the year of research. The digital inequality is mostly analyzed according to ideological attribute (16 of 58) and personal force (14 of 58), both on macro cause. Personal force is continually analyzed according to referenced years, but ideological force started to be analyzed from 2010 until 2020, continually. The educational resource as meso causes and motivational access as micro causes of digital inequalities are the topics of research the most mentioned not specifically at one point of time. 


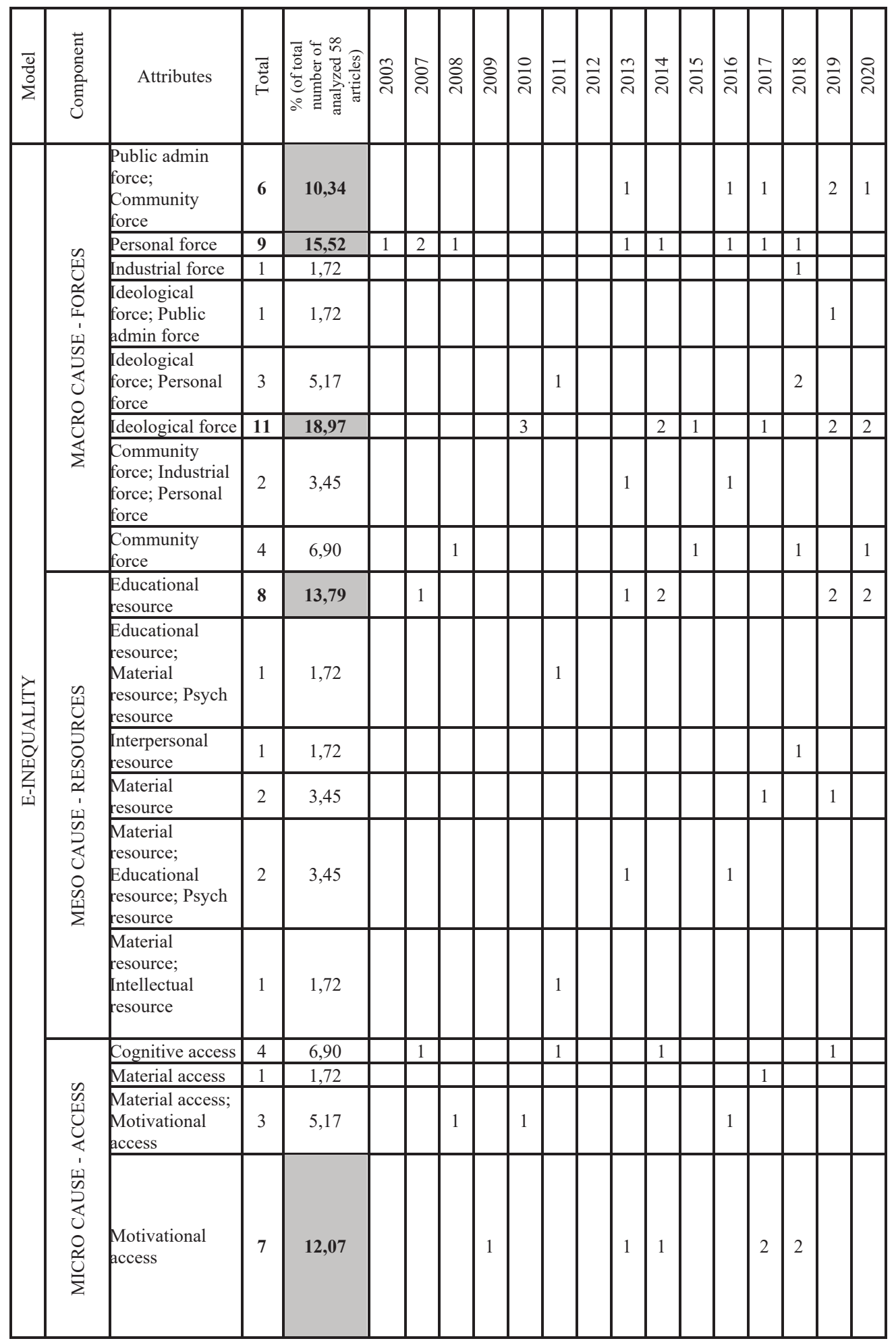




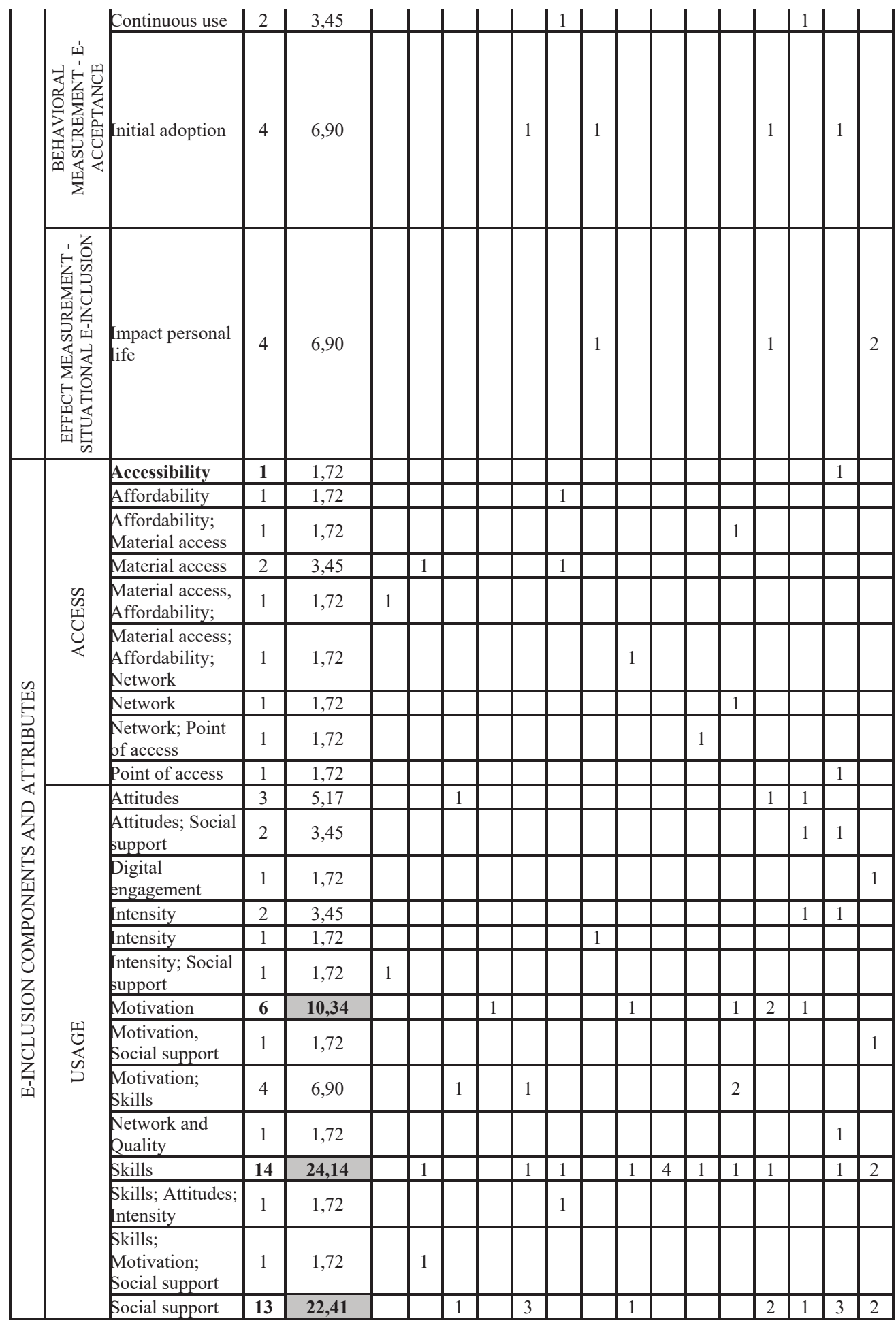




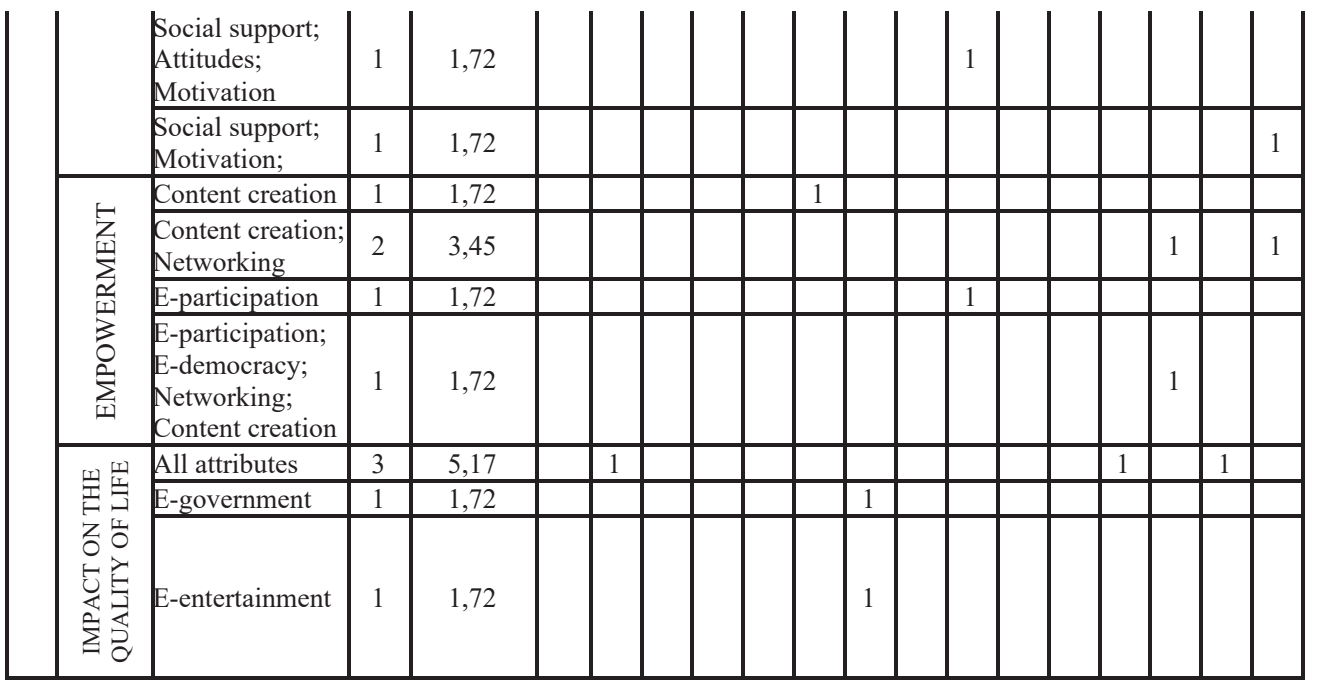

Table 11. Paper by components and attributes in period $2003-2020$.

The skills (20 of 58) and social support (20 of 58) are the most represented attributes of e-Inclusion model. The social support has often been researched in recent years, but skills are continually in the focus of research during observed period. As both are the attributes of usage component, it is clear the usage is the most analyzed component of the e-Inclusion model. Access and empowerment, as well as the impact on the quality of life are rarely being present in sample of analyzed articles.

The analysis showed, among other things, that some papers are related with more than one component of the referenced models (digital inequality or e-Inclusion), which also confirms the multidisciplinary approach of researches. The component macro cause is relatively the most researched component of the digital inequality models in both of papers type (scientific and professional). As it can be concluded from Table 12, the scientific type of papers follows decreasing trend in the meso cause (13), micro cause (11) components. According to selected papers, the focus of scientific papers on behavioral and effect measurement is relatively low. The point is that scientific type of research focuses much more on general macro causes of the inequalities than on the individual adoption and continual use or impact on people's quality of life, which ultimately leads to a successful e-Inclusion process. The professional papers are focused rarely on resources and measurement (behavioral or effect). Thus, it can be said that all components of the model must be equally represented, in order to achieve progressive results of e-Inclusion.

By viewing data in Table 13 it can be seen that scientific papers are focused on usage (46 of 50) and access (11 of 50). In professional papers the focus on the impact on QoL is not observed, but therefore greater focus is given to the component usage. The component impact on QoL is also rarely represented in scientific research (6 of 50). The component empowerment is represented only in 4 scientific papers and 1 professional one. It is relatively negative that these two components are in such a small extent represented in research, because they are the core point of the whole process of 
being e-included for individuals, as well as for other stakeholders (public authorities, business community and other stakeholders).

\begin{tabular}{|l|c|c|c|c|c|}
\hline Type of document & $\begin{array}{c}\text { MACRO CAUSE } \\
\text { - Forces }\end{array}$ & $\begin{array}{c}\text { MESO CAUSE - } \\
\text { Resources }\end{array}$ & $\begin{array}{c}\text { MICRO CAUSE - } \\
\text { Access }\end{array}$ & $\begin{array}{c}\text { BEHAVIORAL } \\
\text { MEASUREMENT - } \\
\text { E-ACCEPTANCE }\end{array}$ & $\begin{array}{c}\text { EFFECT } \\
\text {-SITUAREMENT } \\
\text { E-INCLUSION }\end{array}$ \\
\hline Professional & 7 & 2 & 4 & 1 & 1 \\
\hline Scientific & 30 & 13 & 11 & 6 & 3 \\
\hline Total & 37 & 15 & 15 & 7 & 4 \\
\hline$\%$ & 63,79 & 25,86 & 25,86 & 12,07 & 6,9 \\
\hline
\end{tabular}

Table 12. Paper by type and digital inequality model component.

\begin{tabular}{|l|c|c|c|c|}
\hline Type of document & ACCESS & USAGE & EMPOWERMENT & IMPACT ON QoL \\
\hline Professional & 1 & 7 & 1 & 0 \\
\hline Scientific & 11 & 46 & 4 & 6 \\
\hline Total & 12 & 53 & 5 & 6 \\
\hline$\%$ & 20,69 & 91,38 & 8,62 & 10,34 \\
\hline
\end{tabular}

Table 13. Paper by type and e-Inclusion model component.

Most of analyzed papers consider the digital divide and e-Inclusion as a multidimensional and multiperspective issues as it is presented in Table 14. The total number of keywords defined at the start of content research is 17 . The analysis of papers has resulted in following conclusions:

- $\quad 14 \%$ of scientific papers are related with 9, 6 and 5 keywords; in the group of professional papers, $38 \%$ of them are related with 11 and 8 keywords in the content of papers.

- The professional papers have a wider spectrum of view than the scientific ones and are focused only on specific issues related with a reduced number of referenced keywords in the content.

\begin{tabular}{|l|c|c|c|c|c|c|c|c|c|c|c|c|c|c|}
\hline $\begin{array}{l}\text { Number of related } \\
\text { referenced key words } \\
\text { in the papers' content }\end{array}$ & 14 & 13 & 12 & 11 & 10 & 9 & 8 & 7 & 6 & 5 & 4 & 3 & 2 & Total \\
\hline Scientific & 1 & 2 & 3 & 1 & 5 & 7 & 5 & 5 & 7 & 7 & 4 & 2 & 1 & 50 \\
\hline$\%$ of scientific total & 2 & 4 & 6 & 2 & 10 & 14 & 10 & 10 & 14 & 14 & 8 & 4 & 2 & 100 \\
\hline Professional & 0 & 1 & 0 & 3 & 0 & 0 & 3 & 0 & 0 & 0 & 1 & 0 & 0 & 8 \\
\hline$\%$ of professional total & 0 & 12,5 & 0 & 38 & 0 & 0 & 38 & 0 & 0 & 0 & 12,5 & 0 & 0 & 100 \\
\hline
\end{tabular}

Table 14. Papers by number of related referenced key words in the papers' content.

The authors have also analyzed potential future research suggestions from selected scientific papers indexed in Scopus or in WoS (40 of 58). Seven of 40 papers contain the exact recommendations and future work ideas, as it stands in Table as follows. 


\begin{tabular}{|c|c|}
\hline Reference of the document & Recommendations and future work ideas \\
\hline $\begin{array}{c}\text { Zhang, X., Tlili, A., } \\
\text { Nascimbeni, F. et al. } \\
\text { Accessibility within open } \\
\text { educational resources and } \\
\text { practices for disabled learners: } \\
\text { a systematic literature review. } \\
\text { Smart Learn. Environ. 7, } 1 \\
\text { (2020) }\end{array}$ & $\begin{array}{l}\text { This study opens new research perspectives for } \\
\text { researchers and practitioners on the use of open } \\
\text { educational resources and practices for accessibility and } \\
\text { functional diversity in educational contexts by uncovering } \\
\text { gaps in this field that should be investigated. }\end{array}$ \\
\hline $\begin{array}{l}\text { Morris, A., Goodman, J., \& } \\
\text { Brading, H. (2007) }\end{array}$ & $\begin{array}{l}\text { There is much to be done to change the perceptions of } \\
\text { older people and to provide } \\
\text { facilities more suited to their use. }\end{array}$ \\
\hline $\begin{array}{l}\text { Boulton-Lewis, G. M., Buys, } \\
\text { L., Lovie-Kitchin, J., Barnett, } \\
\text { K., \& David, L. N. (2007) }\end{array}$ & $\begin{array}{l}\text { The need for access to computers for people with lower } \\
\text { incomes also needs to be addressed. }\end{array}$ \\
\hline $\begin{array}{r}\text { van Deursen } \\
\qquad(20\end{array}$ & $\begin{array}{l}\text { Administrators are responsible for the institution of the } \\
\text { public information supply, both internally and externally. } \\
\text { They have to decide on the acquisition and } \\
\text { implementation of infrastructures, architectures and } \\
\text { applications and assess whether these fit within the } \\
\text { existing organization or whether they need to be adapted. } \\
\text { It is therefore recommended that administrators possess } \\
\text { more Internet skills. Policy advisors should support } \\
\text { administrators in decision-making and have to be aware } \\
\text { of all possibilities that the Internet offers to the } \\
\text { government. It is highly recommended to improve the } \\
\text { levels of Internet skills among civil } \\
\text { servants, especially the levels of information and strategic } \\
\text { Internet skills; It is recommended that the Internet skill } \\
\text { levels are tested when hiring new employers, preferably } \\
\text { using tests or surveys. }\end{array}$ \\
\hline $\begin{array}{l}\text { Bejaković, P., \& Mrnj } \\
\text { (2020) }\end{array}$ & $\begin{array}{l}\text { The government, educational institutions and employers } \\
\text { should design new forms to assess digital skills, change } \\
\text { standards to reflect the value of } 21 \text { st century literacy, } \\
\text { design and implement intervention programs for } \\
\text { workforce digital skills development. For all these } \\
\text { demanding tasks, there is a need for a strong partnership } \\
\text { on the national and European level, where stakeholders } \\
\text { work together to reduce the digital skills gap. }\end{array}$ \\
\hline $\begin{array}{l}\text { Martínez-Bravo, M. C., } \\
\text { Sádaba-Chalezquer, C., \& } \\
\text { Serrano-Puche, J. (2020) }\end{array}$ & $\begin{array}{l}\text { It is also important to point out that throughout the } \\
\text { research process and data processing we identified other } \\
\text { terms of interest that have been left out of the analysis } \\
\text { such as: computer literacy, e-literacy, internet literacy, } \\
\text { among others, which should be considered in future } \\
\text { studies. }\end{array}$ \\
\hline
\end{tabular}

Table 15. Recommendations from selected scientific researches. 
Ten of 40 selected papers have specified the limitations related to those researches. The limitations of scientific researches like reduced number of criteria parameters such as keywords, sample size, population groups, specific factors important for research issues, variables and geographic territory were included in the research.

Future work was specified in eleven of 40 papers, and is mostly related with previously mentioned limitations of the research by providing research on a bigger sample, on wider geographic territory by using more variables or factors important for research topics, including wider population, etc.

Regarding the criteria that was used in this research and two referenced models that presents the backbone of the research methodology it is reviled that e-Inclusion and digital divide attributes of the models did not attracted attention of the researches by the same intensity. The impact on quality of life is not enough emphasized as the motivational factor for raising e-Inclusion of vulnerable population groups. The developed countries have much more research related with key issues and the most important is that they recognized the importance much earlier of that and take practical measures according the public policies to make thing better. So the results of that are visible according the results of e-society research conducted by EUROSTAT.

\section{Conclusion}

The main contribution of this paper is that it connects two referenced models - the multidimensional and multiperspective theoretical model of e-Inclusion [65] and integrated model of digital inequality [32] by focusing on keywords of analyzed documents. The main idea was to make the landscape of research during the period of 2003-2020 and find out which components of actual referenced models are emphasized. The problem of digital divide is still present, which means the focus of researchers and practitioners must be moved to those components of referenced models that can assure the potential raise of e-Inclusion regarding the attitudes of all kind of e-Inclusion vulnerable social groups. It means that practitioners have to use the scientific results more in the process of making programs for e-Inclusion of vulnerable social groups. According the goal of this research the article presents the crosscut of the causes of the digital divide and effects of e-Inclusion.

The paper presents the bridge between digital divide and e-Inclusion, and keywords are bottleneck for raising e-Inclusion.

According to the results presented in this study, several concluding remarks could be specified:

- The authors of analyzed papers in their research have been mostly focused on components usage and access, and less on components empowerment and impact on QoL. Empowerment and impact on QoL are more associated with motivational issues and both have a significant impact on raising the eInclusion rate. It could be concluded that the impacts of e-Inclusion on quality of life are not presented enough as motivational mechanisms to raise the einclusion rate of vulnerable population groups.

- The causes of digital inequalities are relatively analyzed much more than the behavioral measurement of e-acceptance and effect measurement of 
situational e-Inclusion. This is a similar situation to the previous note, but from the point of practice that follows the scientific conclusions, it could be said that the effect measurement of situational e-Inclusion or the behavioral measurement of e-acceptance should be seen as motivational factor for raising e-Inclusion rate in global.

\section{Limitations and future work}

Regarding the results of presented research, especially those related with bridging two referenced models by keywords from selected papers, the authors of this research can specify few limitations. The limitation of this paper is that the documents considered are in English language. Also, it has to be mentioned that multidimensional and multiperspective theoretical model of e-Inclusion has much more attributes than the analyzed keywords which are taken into consideration during research - like the attributes which together form the component impact of quality of life (e-government, e-health, e-learning...). These attributes are key components of digital society developing processes - and in nature they are a kind of digital services that are the objects of usage and - in authors' opinion - must be separately analyzed from that aspect, which also represents the idea for the future research.

Future work will focus more on components empowerment and raising quality of life by e-Inclusion of vulnerable social groups. Until now, that was recognized by public administration and politicians for distribution of public information of their work. Citizens have to use much more among all e-services that have positive impact on quality of life, so the future research will be oriented to those attributes referenced in e-Inclusion model [65].

\section{Appendix 1. Analyzed scientific and professional papers}

\begin{tabular}{|c|c|}
\hline $\begin{array}{c}\text { Order } \\
\text { No. }\end{array}$ & List of analyzed scientific and professional papers \\
\hline 1. & $\begin{array}{l}\text { A. Lima Oliveira, et.al., "Promoting conscious and active learning and aging - } \\
\text { How to face current and future challenges?", available on } \\
\text { https://www.uc.pt/imprensa_uc/catalogo/ebook/E-book_Promoting, } 2013 .\end{array}$ \\
\hline 2. & $\begin{array}{l}\text { G. Galdon Clavell, M.M. Zamorano, J.M. Zavala Pérez, "ICTs and Community } \\
\text { Policing: An Ethical Framework. In: Leventakis G., Haberfeld M. (eds)", Societal } \\
\text { Implications of Community-Oriented Policing and Technology", pp 63-76. } \\
\text { SpringerBriefs in Criminology. Springer, Cham, https://doi.org/10.1007/978-3- } \\
\text { 319-89297-9_8, 2018. }\end{array}$ \\
\hline 3. & $\begin{array}{l}\text { A.C. Kirongo, G.S. Huka, D.G. Bundi, G.M. Muketha, "Competence Network for } \\
\text { e-Inclusion and Assistive Technologies", available on } \\
\text { http://www.iosrjournals.org/iosr-jhss/papers/Vol.\%2024\%20Issue6/Series- } \\
\text { 7/H2406076670.pdf, } 2019 \text {. }\end{array}$ \\
\hline 4. & $\begin{array}{l}\text { E. Brenna, "Adult Education, the Use of Information and Communication } \\
\text { Technologies and Their Impact on Elderly's Quality of Life: A Case Study", } \\
\text { International Journal of Business and Social Science, 10(8), } 2019 .\end{array}$ \\
\hline
\end{tabular}




\begin{tabular}{|c|c|}
\hline 5. & $\begin{array}{l}\text { O. Sergeyeva \& L. Makarova, "E-Inclusion and perception of time among } \\
\text { elderly: Russian regional perspective", International Journal of Electronic } \\
\text { Governance, 8(3), 303-313, } 2016 .\end{array}$ \\
\hline 6. & $\begin{array}{l}\text { X. Zhang, A. Tlili, F. Nascimbeni, et al., "Accessibility within open educational } \\
\text { resources and practices for disabled learners: a systematic literature review", } \\
\text { Smart Learn. Environ. 7, 1, https://doi.org/10.1186/s40561-019-0113-2,2020. }\end{array}$ \\
\hline 7. & $\begin{array}{l}\text { N. Selwyn, S. Gorard, J. Furlong \& L. Madden, "Older adults' use of information } \\
\text { and communications technology in everyday life", Ageing and society, 23, 561, } \\
2003 .\end{array}$ \\
\hline 8. & $\begin{array}{l}\text { A. Morris, J. Goodman \& H. Brading, "Internet use and non-use: views of older } \\
\text { users", Universal access in the information society, } 6(1), 43-57,2007 .\end{array}$ \\
\hline 9. & $\begin{array}{l}\text { G. M. Boulton-Lewis, L. Buys, J. Lovie-Kitchin, K. Barnett \& L.N. David, } \\
\text { "Ageing, learning, and computer technology in Australia", Educational } \\
\text { Gerontology, 33(3), 253-270, } 2007 .\end{array}$ \\
\hline 10. & $\begin{array}{l}\text { R-A. Petrauskas, T. Bilevičienė, A. Kiškienė, "E-Inclusion as a Part of E- } \\
\text { Government Developlemnt in Lithuania", ISSN 1648-260, Viešoji Politika Ir } \\
\text { adMINISTRAVIMS, } 2008 \text { Nr. } 23 \text { https://www3.mruni.eu/ojs/public-policy-and- } \\
\text { administration/article/view/2011/1821, 2008. }\end{array}$ \\
\hline 11. & $\begin{array}{l}\text { A. Siren \& L. Hakamies-Blomqvist, "Mobility and well-being in old age.", Topics } \\
\text { in Geriatric Rehabilitation, 25(1), 3-11, } 2009 .\end{array}$ \\
\hline 12 . & $\begin{array}{l}\text { A. van Deursen, J. van Dijk, "Civil Servants' Internet Skills: Are They Ready for } \\
\text { E-Government?. In: Wimmer M.A., Chappelet JL., Janssen M., Scholl H.J. } \\
\text { (eds)", Electronic Government pp 132-143. EGOV 2010. Lecture Notes in } \\
\text { Computer Science, vol 6228. Springer, Berlin, Heidelberg, } \\
\text { https://doi.org/10.1007/978-3-642-14799-9_12,2010. }\end{array}$ \\
\hline 13. & $\begin{array}{l}\text { D. Epstein, E.C. Nisbet \& T. Gillespie, "Who's responsible for the digital divide? } \\
\text { Public perceptions and policy implications.", The Information Society, 27(2), 92- } \\
104,2011 .\end{array}$ \\
\hline 14. & $\begin{array}{l}\text { W. Vishanth, Y.K. Dwivedi, R. El-Haddadeh, A. Almuwil \& A. Ghoneim, } \\
\text { "Conceptualizing E-Inclusion in Europe: An Explanatory Study", Information } \\
\text { Systems Management, 29:4, 305-320, DOI: 10.1080/10580530.2012.716992, } \\
2012\end{array}$ \\
\hline 15. & $\begin{array}{l}\text { M. Näsi, P. Räsänen \& O. Sarpila, "ICT activity in later life: Internet use and } \\
\text { leisure activities amongst senior citizens in Finland", European Journal of } \\
\text { Ageing, 9(2), 169-176., 2012. }\end{array}$ \\
\hline 16. & $\begin{array}{l}\text { B. Rerup Schlichter, L. Danylchenko, "Measuring ICT usage quality for } \\
\text { information society building", Government Information Quarterly Volume 31, } \\
\text { Issue 1, January 2014, Pages 170-184, https://doi.org/10.1016/j.giq.2013.09.003, } \\
2014 \text {. }\end{array}$ \\
\hline 17. & $\begin{array}{l}\text { S. Bannier, R. Glott., V. Meijs, "How E-Inclusion and Innovation Policy Affect } \\
\text { Digital Access and Use for Senior Citizens in Europe. In: Stephanidis C., Antona } \\
\text { M. (eds) Universal Access in Human-Computer Interaction. User and Context } \\
\text { Diversity. UAHCI 2013", Lecture Notes in Computer Science, vol 8010. Springer, } \\
\text { Berlin, Heidelberg. https://doi.org/10.1007/978-3-642-39191-0_1, 2013. }\end{array}$ \\
\hline 18. & $\begin{array}{l}\text { H. Ayako, K. Masaaki, "Active Use of ICTs among the Elderly by Positive User } \\
\text { Experience", International Journal of Computer Science and Information } \\
\text { Security, Vol. 11, No. 3, 2013. http://sites.google.com/site/ijcsis, ISSN 1947- } \\
5500,2013 \text {. }\end{array}$ \\
\hline
\end{tabular}




\begin{tabular}{|c|c|}
\hline 19. & $\begin{array}{l}\text { A. Loureiro, M. Barbas, "Active Ageing - Enhancing Digital Literacies in Elderly } \\
\text { Citizens. In: Zaphiris P., Ioannou A. (eds) Learning and Collaboration } \\
\text { Technologies. Technology-Rich Environments for Learning and Collaboration pp } \\
\text { 450-459. LCT 2014.", Lecture Notes in Computer Science, vol 8524. Springer, } \\
\text { Cham, https://doi.org/10.1007/978-3-319-07485-6 44, 2014. }\end{array}$ \\
\hline 20. & $\begin{array}{l}\text { J. Billestrup, J. Stage, "E-government and the Digital Agenda for Europe. In: } \\
\text { Marcus A. (eds) Design, User Experience, and Usability. User Experience Design } \\
\text { for Diverse Interaction Platforms and Environments, pp 71-80. DUXU 2014.", } \\
\text { Lecture Notes in Computer Science, vol 8518. Springer, Cham, } \\
\text { https://doi.org/10.1007/978-3-319-07626-3 7, 2014. }\end{array}$ \\
\hline 21. & $\begin{array}{l}\text { I. Watkins, B. Xie, "eHealth literacy interventions for older adults: a systematic } \\
\text { review of the literature", Journal of medical Internet research, 16(11), e225, } \\
2014 .\end{array}$ \\
\hline 22 & $\begin{array}{l}\text { F. Comunello, S. Mulargia, F. Belotti, M. Fernández-Ardèvol, "Older People’s } \\
\text { Attitude Towards Mobile Communication in Everyday Life: Digital Literacy and } \\
\text { Domestication Processes. In: Zhou J., Salvendy G. (eds) Human Aspects of IT for } \\
\text { the Aged Population. Design for Aging, pp 439-450. ITAP 2015.", Lecture Notes } \\
\text { in Computer Science, vol 9193. Springer, Cham, https://doi.org/10.1007/978-3- } \\
\text { 319-20892-343, 2015. }\end{array}$ \\
\hline 23. & $\begin{array}{l}\text { E. Chalkia, E. Bekiaris, R.I. Madrid, "Evaluating All-Inclusive ICT with } \\
\text { Developers, End Users and Stakeholders. In: Zhou J., Salvendy G. (eds) Human } \\
\text { Aspects of IT for the Aged Population. Design for Aging, pp 157-165. ITAP } \\
\text { 2015.", Lecture Notes in Computer Science, vol 9193. Springer, Cham, } \\
\text { https://doi.org/10.1007/978-3-319-20892-3_16, 2015. }\end{array}$ \\
\hline 24. & $\begin{array}{l}\text { R. Sharma, A.R. Fantin, N. Pra } \\
\text { knowledge societies: A ground } \\
\text { development", Telecommunicat }\end{array}$ \\
\hline 25 & $\begin{array}{l}\text { A. Berenguer, J. Goncalves, S. Hosio, D. Ferreira, T. Anagnostopoulos, V. } \\
\text { Kostakos, "Are smartphones ubiquitous?: An in-depth survey of smartphone } \\
\text { adoption by seniors", IEEE Consumer Electronics Magazine, 6(1), 104-110, } \\
2016 .\end{array}$ \\
\hline 26. & $\begin{array}{l}\text { T.N. Friemel, "The digital divide has grown old: Determinants of a digital divide } \\
\text { among seniors", New media \& society, 18(2), 313-331, } 2016 \text {. }\end{array}$ \\
\hline 27. & $\begin{array}{l}\text { M. Viñarás-Abad, L. Abad-Alcalá, C. Llorente-Barroso, M. Sánchez-Valle, M. } \\
\text { Pretel-Jiménez, “e-Administration and the e-Inclusion of the elderly”. Revista } \\
\text { Latina de Comunicación Social, 72, pp. } 197 \text { to 219., } \\
\text { http://www.revistalatinacs.org/072paper/1161/11en.html, DOI: 10.4185/RLCS- } \\
\text { 2017-1161en, } 2017\end{array}$ \\
\hline 28. & $\begin{array}{l}\text { H. Dudek, W. Szczesny, "Correlates of Multidimensional Indicator of Quality of } \\
\text { Life-Fractional Outcome Model Approach", STATISTIKA-STATISTICS AND } \\
\text { ECONOMY JOURNAL, 97(4), 45-60, 2017. }\end{array}$ \\
\hline 29. & $\begin{array}{l}\text { A. Siren, S.G. Knudsen, "Older adults and emerging digital service delivery: A } \\
\text { mixed methods study on information and communications technology use, skills, } \\
\text { and attitudes", Journal of aging \& social policy, 29(1), 35-50, } 2017 \text {. }\end{array}$ \\
\hline 30. & $\begin{array}{l}\text { F. Ruiz-Rodríguez, A.L. Lucendo-Monedero, R. González-Relaño, "Measurement } \\
\text { and characterisation of the Digital Divide of Spanish regions at enterprise level. A } \\
\text { comparative analysis with the European context", Telecommunications Policy, }\end{array}$ \\
\hline
\end{tabular}




\begin{tabular}{|c|c|}
\hline & $\begin{array}{l}\text { Volume 42, Issue 3, Pages 187-211, ISSN 0308-5961, } \\
\text { https://doi.org/10.1016/j.telpol.2017.11.007, 2018. }\end{array}$ \\
\hline 31. & $\begin{array}{l}\text { P. Chipeva, F. Cruz-Jesus, T. Oliveira, Z. Irani, "Digital divide at individual level: } \\
\text { Evidence for Eastern and Western European countries", Government Information } \\
\text { Quarterly, 35(3), 460-479., DOI: 10.1016/j.giq.2018.06.003, } 2018 .\end{array}$ \\
\hline 32. & $\begin{array}{l}\text { J. Trilar,A. Kos, S. Jazbinšek, L. Jensterle, E. Stojmenova Duh, "ICT to Promote } \\
\text { Well-Being within Families", Sensors, 18(9), 2760, } 2018 .\end{array}$ \\
\hline 33. & $\begin{array}{l}\text { A. Al-Muwil, V. Weerakkody, R. El-haddadeh, et al. "Balancing Digital-By- } \\
\text { Default with Inclusion: A Study of the Factors Influencing E-Inclusion in the } \\
\text { UK", Inf Syst Front 21, 635-659, https://doi.org/10.1007/s10796-019-09914-0, } \\
\text { 2019. }\end{array}$ \\
\hline 34. & $\begin{array}{l}\text { S. Atarodi, A.M. Berardi, A-M. Toniolo, "Comparing local policy practicesto } \\
\text { implement ICT-based home care services for aging-in-place in Finland, France, } \\
\text { Italy, Spain\& Sweden", Gerontechnology, ISG International Society for } \\
\text { Gerontechnology, 2019, } 18 \text { (2), 10.4017/gt.2019.18.2.005.00 . hal-02315164, } \\
2019 .\end{array}$ \\
\hline 35. & $\begin{array}{l}\text { L. Moreno, P. Martinez, "The harmonization of accessibility standards for public } \\
\text { policies", Computer, 52(7), 57-66, } 2019 \text {. }\end{array}$ \\
\hline 36. & $\begin{array}{l}\text { I. Gal, A. Grotlüschen, D. Tout, G. Kaiser, "Numeracy, adult education, and } \\
\text { vulnerable adults: a critical view of a neglected field", ZDM Mathematics } \\
\text { Education, https://doi.org/10.1007/s11858-020-01155-9, } 2020 .\end{array}$ \\
\hline 37. & $\begin{array}{l}\text { P. Bejaković, Ž. Mrnjavac, "The importance of digital literacy on the labour } \\
\text { market", Employee Relations: The International Journal , } 2020 .\end{array}$ \\
\hline 38. & $\begin{array}{l}\text { J. Guenther, B. Smede, M. Young, "Digital inclusion in central Australia: what is } \\
\text { it and what makes it different?", Rural Society, 1-17, } 2020 .\end{array}$ \\
\hline 39. & $\begin{array}{l}\text { M. C. Martínez-Bravo, C. Sádaba-Chalezquer, J. Serrano-Puche, "Fifty years of } \\
\text { digital literacy studies: A meta-research for interdisciplinary and conceptual } \\
\text { convergence", Profesional de la Información, 29(4), } 2020 .\end{array}$ \\
\hline 40 & $\begin{array}{l}\text { X. Sun, W. Yan, H. Zhou, Z. Wang, X. Zhang, S. Huang, S., L. Li, " Internet use } \\
\text { and need for digital health technology among the elderly: a cross-sectional survey } \\
\text { in China", BMC public health, 20(1), 1-8, 2020. }\end{array}$ \\
\hline 41. & $\begin{array}{l}\text { P. Silva, AD. Matos, R. Martinez-Pecino, "E-Inclusion: Beyond individual socio- } \\
\text { demographic characteristics", PLoS ONE 12(9):e0184545. } \\
\text { https://doi.org/10.1371/journal., pone.0184545, } 2017 .\end{array}$ \\
\hline 42 & $\begin{array}{l}\text { European Social Survey (ESS), "Exploring public attitudes, informing public } \\
\text { policy: Selected findings from the first three rounds.", ESS, } \\
\text { https://ess.sciencespo.com/wp- } \\
\text { content/uploads/sites/16/2017/08/ESSFindingsBooklet.pdf, } 2008 \text {. }\end{array}$ \\
\hline 43. & $\begin{array}{l}\text { D. Leahy, D. Dolan, "Digital Literacy: A Vital Competence for 2010? In: } \\
\text { Reynolds N., Turcsányi-Szabó M. (eds) Key Competencies in the Knowledge } \\
\text { Society.", KCKS, pp 210-221. IFIP Advances in Information and Communication } \\
\text { Technology, vol 324. Springer, Berlin, Heidelberg, https://doi.org/10.1007/978-3- } \\
\text { 642-15378-5_21, ISSN: 1868-4238, 2010. }\end{array}$ \\
\hline 44. & $\begin{array}{l}\text { U. Maier-Rabler, "E-Policies and the Diversity of European Information } \\
\text { Cultures", European Sociological Association Research Network Sociology of } \\
\text { Culture Midterm Conference: Culture and the Making of Worlds, October 2010, } \\
\text { Available at SSRN: https://ssrn.com/abstract }=1692198,2010 \text {. }\end{array}$ \\
\hline
\end{tabular}




\begin{tabular}{|c|c|}
\hline 45. & $\begin{array}{l}\text { B. Bunker, "A summary of international reports, research, and case studies of } \\
\text { digital literacy", New Zealand Computer Society, Inc. } \\
\text { https://www.scribd.com/document/385623431/201001-Digital-Literacy- } \\
\text { Research-Report, 2010. }\end{array}$ \\
\hline 46. & $\begin{array}{l}\text { M. Haasjes, www.iageproject.eu, } \\
\text { http://archive.northsearegion.eu/files/repository/20141216164843_ROM\&N1206 } \\
\text { 0602-iAgefolder(LR).pdf, 2014. }\end{array}$ \\
\hline 47. & $\begin{array}{l}\text { G. Nistor, "New educational strategies regarding quality of life for elderly } \\
\text { people", Procedia-Social and Behavioral Sciences, 142, 487-492, } 2014 .\end{array}$ \\
\hline 48. & $\begin{array}{l}\text { L.A. Alcalá, "Media literacy for older people facing the digital divide: The e- } \\
\text { Inclusion programmes design", Comunicar. Media Education Research } \\
\text { Journal, 22(1), } 2014 \text {. }\end{array}$ \\
\hline 49. & $\begin{array}{l}\text { D. Padilla-Góngora, R. López-Liria, M. del Pilar Díaz-López, J.M. Aguilar-Parra, } \\
\text { M.E. Vargas-Muñoz, P. Rocamora-Pérez, "Habits of the elderly regarding access } \\
\text { to the new information and communication technologies", Procedia-Social and } \\
\text { Behavioral Sciences, 237, 1412-1417, } 2017 .\end{array}$ \\
\hline 50. & $\begin{array}{l}\text { Centre for Economics and Business Research (Cebr), } \\
\text { https://www.goodthingsfoundation.org/research-publications/economic-impact- } \\
\text { digital-inclusion-uk, } 2018 \text {. }\end{array}$ \\
\hline 51. & $\begin{array}{l}\text { S. Davidson, "Digital Inclusion Evidence Review 2018", available on } \\
\text { https://www.ageuk.org.uk/globalassets/age-uk/documents/reports-and- } \\
\text { publications/age_uk_digital_inclusion_evidence_review_2018.pdf, } 2018 .\end{array}$ \\
\hline 52. & $\begin{array}{l}\text { C. Richards, "Later life learning from experience: the cross-cultural importance of } \\
\text { 'life reviews' in seniors' lifelong education and learning", ZfW 42, 5-22 . } \\
\text { https://doi.org/10.1007/s40955-018-0123-7, 2019. }\end{array}$ \\
\hline 53. & $\begin{array}{l}\text { C. Burr, M. Taddeo, L. Floridi, "The Ethics of Digital Well-Being: A Thematic } \\
\text { Review", Sci Eng Ethics, https://doi.org/10.1007/s11948-020-00175-8, } 2020 .\end{array}$ \\
\hline 54. & $\begin{array}{l}\text { European Commission, "ICT Research The policy perspective. ICT for all, } \\
\text { Technology supporting an inclusive world", available on } \\
\text { https://cordis.europa.eu/ictresults/pdf/policyreport/INF\%207\%200100\%20IST- } \\
\text { R\%20policy\%20report-eInclusion final\%20studio.pdf, } 2010 \text {. }\end{array}$ \\
\hline 55. & $\begin{array}{l}\text { V. Dolničar, M. Setinc, "Digital inclusion and active ageing - developing a user- } \\
\text { centred methodological approach to investigate the use of mobile phones among } \\
\text { older people - Good practice project", available on } \\
\text { https://www.interregeurope.eu/fileadmin/user_upload/tx_tevprojects/library/file_ } \\
\text { 1501845343.pdf,2017. }\end{array}$ \\
\hline 56. & $\begin{array}{l}\text { F. Arrieta, "The European Social Model. In: Gu D., Dupre M. (eds) Encyclopedia } \\
\text { of Gerontology and Population Aging", Springer, Cham. } \\
\text { https://doi.org/10.1007/978-3-319-69892-2_518-1,2019. }\end{array}$ \\
\hline 57. & $\begin{array}{l}\text { M. Repetto, G. Trentin, "in IFIP International Federation for Information } \\
\text { Processing, Volume 281; Learning to Live in the Knowledge Society; Michael } \\
\text { Kendall and Brian Samways", Springer, pp. 279-286, } 2008 .\end{array}$ \\
\hline 58. & $\begin{array}{l}\text { A. Almuwil, V. Weerakkody, R. El-Haddadeh, "A Conceptual Study of the } \\
\text { Factors Influencing e-Inclusion", European, Mediterranean \& Middle Eastern } \\
\text { Conference on Information Systems 2011(EMCIS2011) May 30-31 2011, } \\
\text { Athens,Greece, https://bura.brunel.ac.uk/bitstream/2438/8504/2/Fulltext.pdf, } \\
2011 .\end{array}$ \\
\hline
\end{tabular}




\section{Acknowledgement}

The paper has been prepared in the context of the Twinning Open Data Operational (TODO) project which has received funding from the European Union's Horizon 2020 research and innovation programme under Grant Agreement Number 857592 TODO.

\section{References}

[1] J. Binde, "Towards knowledge societies: UNESCO World Report," Paris: UNESCO Publishing, 2005.

[2] J. Van Dijk, "The network society. Social aspects of new media" Thousand Oaks. CA: Sage. London. UK. 1999

[3] J. Van Dijk, and A.G.M. "The Deepening Divide. Inequality in the Information Society," Thousand Oaks. CA: Sage. London. UK. (2005)

[4] J. Van Dijk, "Digital divide research, achievements and shortcomings". Poetics. Vol.34(4-5), 221-235., 2006.

[5] J. Van Dijk, W. Pieterson, W. Ebbers, and A. Van Deursen, "eServices for Citizens: TheDutch Usage Case," In: Wimmer, M.A., Scholl, J., Grönlund, A. (eds.) EGOV 2007. LNCS. Springer, Heidelberg, 4656, 155-166., 2007.

[6] P. DiMaggio and E. Hargittai, "From the 'digital divide' to 'digital inequality': Studying Internet use as penetration increase (Working Paper 19)," Princeton, NJ: Center for Artsand Cultural Policy Studies, Woodrow Wilson School, Princeton University, 2001. [Online]. Available: http://www.princeton.edu/wartspol/workpap/WP15\%20\%20 DiMaggio\%2BHargittai.pdf. [Accessed July 16, 2013].

[7] P. Norris, "Digital divide: Civic engagement, information poverty, and the Internet worldwide," Cambridge University Press. London. UK. 2001.

[8] E. Mordini, D. Wright, P. De Hert, E. Mantovani, K. R. Wadhwa, J. Thestrup, and G. Van Steendam, "Ethics, e-Inclusion and ageing," Studies in Ethics, Law, and Technology. Vol. 3(1), 2009. [Online]. Available: http://www.researchgate.ne t/profile/Emilio_ Mor dini/publication/40823126_Ethics_eInclusion_and_Ageing/links/02bfe511 e76cc0c589000000.pdf [Accessed October 26, 2013]

[9] National Telecommunications and Information Administration (NTIA) "Falling Through the Net II: New Data on the Digital Divide," 1998. [Online]. Available: http://www. ntia.doc.gov/ntiahome/net2; http://www.ntia.doc.gov/files/ntia/publications/ falling-through-net-ii.pdf [Accessed August 17, 2014]. 
[10] OECD, "Understanding the digital divide. Paris: OECD," 2001. Available: http://www.oecd.org/dataoecd/38/57/1888451.pdf [Accessed July 17, 2013.]

[11] S. Dewan, and F.J. Riggins, "The Digital Divide: Current and Future Research Directions," Journal of the Association for the Information Systems. Vol. 6(12), 298-337., 2005.

[12] J. Binde, "Towards knowledge societies: UNESCO World Report," Paris: UNESCO Publishing. 2005.

[13] M. Warschauer, "Technology and school reform: a view from both sides of the track," Education Policy Analysis Archives. Vol. 8(4), 1-22., 2000.

[14] M. Warschauer, "Technology and Social Inclusion: Rethinking the Digital Divide," Cambridge: MIT Press. USA., 2003.

[15] I. Meyer, S., Muller, and L., Kubitscheke, "eInclusion - Towards a Coherent European Policy to Social Inequalities in the Information Society," 2006., [Online]. Available:

http://www.empirica.com/themen/einclusion/documents/Meyer-Muller-ea eInclusion_eChallenges-2006.pdf [Accessed January 07, 2013.].

[16] S. Judge, K. Puckett, and S. M. Bell, "Closing the digital divide: update from the early childhood longitudinal study," Journal of Educational Research. Vol. 100(1), 52-60., 2006.

[17] Y. Li, and M., Ranieri, “Are 'digital natives' really digitally competent? A study on Chinese teenagers," British Journal of Educational Technology. Vol. 41(6), 1029-1042., 2010.

[18] L. Zhao, Y. Lu, W. Huang, and Q. Wang, "Internet inequality: the relationship between high school students' Internet use in different locations and their Internet self efficacy," Computers \& Education. Vol. 55, 1405-1423., 2010.

[19] S. Molnár, "The explanation frame of the digital divide," Proceedings of the Summer School. Risks and Challenges of the Network Society, 4-8. 2003.

[20] International Telecommunication Union (ITU), "Measuring digital opportunity. Paper presented at the WSIS Thematic Meeting on MultiStakeholder Partnerships for Bridging the Digital Divide”, 2005. Available: http://www. itu.int/itu wsis/2005/DOI\%20V2.pdf [Accessed May 14, 2013.]

[21] E. Mancinelli, "e-Inclusion in the Information Society, Information Society From Theory to Political Practice," 2008. Available: http://www.ittk.hu/netis/doc/NETIS_CCourse_Book_English.pdf [Accessed January 7, 2013.] 
[22] O. Kolesnichenko, L. Mazelis, A. Sotnik, D. Yakovleva, S. Amelkin, I. Grigorevsky, and Y. Kolesnichenko, "Sociological modeling of smart city with the implementation of UN sustainable development goals," Sustainability Science, 1-19. 2020.

[23] S. Tsekea, and J. P. Chigwada, "COVID-19: strategies for positioning the university library in support of e-learning," Digital Library Perspectives., 2020.

[24] M. Kaess, M, Moessner, J. Koenig, S. Lustig, S. Bonnet, K. Becker, and ProHEAD Consortium, "A plea for the sustained implementation of digital interventions for young people with mental health problems in the light of the COVID-19 pandemic," Journal of Child Psychology and Psychiatry, 2020

[25] D.J. Wake, F. W. Gibb, P. Kar, B. Kennon, D.C. Klonoff, G. Rayman, and R.K. Semple, "Endocrinology in the time of COVID-19: Remodelling diabetes services and emerging innovation," European Journal of Endocrinology, 183(2), G67-G77., 2020.

[26] B. Pauli Medeiros, L.R. Franco Goldoni, E., Batista Junior, and H. Ribeiro da Rocha, "The use of cyberspace by the public administration in the COVID-19 pandemic: diagnosis and vulnerabilities," RAP: Revista Brasileira de Administração Pública, 54(4), 2020.

[27] D. Mehta, and X. Wang, "COVID-19 and digital library services-a case study of a university library," Digital Library Perspectives, 2020.

[28] EUROSTAT [Online] Available on https:/ec.europa.eu/eurostat/statisticsexplained/index.php/Glossary, last update on 26/01/2021, [Accessed February 10, 2021]

[29] S. Mendonca, N. Crespo, N. Simoes "Inequality in the network society: An integrated approach to ICT access, basic skills, and complex capabilities", Telecommunications Policy, Volume 39, Issues 3-4, May 2015, Pages 192-207, doi.org/10.1016/j.telpol.2014.12.010

[30] T. Huesing, H. Selhofer ,The Digital Divide Index - A Measure of Social Inequalities in the Adopritioon of ICT", 2002. European Conference of Information Systems, 2002, preuzeto 10.12.2017., Proceedings http://aisel.aisnet.org/ecis2002

[31] D. Epstein, E.C. Nisbet, T. Gillespie "Who's Responsible for the Digital Divide? Public Perceptions and Policy Implications", The Information Society, 2011, DOI: 10.1080/01972243.2011.548695

[32] B. Yu, A. Ndumu, L. M. Mon and Z. Fan "E-Inclusion or digital divide: an integrated model of digital inequality", Journal of Documentation, Vol. 74 Issue: 3, pp.552-574, 2018., https://doi.org/10.1108/JD-10-2017-0148 
[33] United Nations ,Transforming our World: The 2030 Agenda for Sustainable Development" A/RES/70/1 , 2015., preuzeto 01.08.2018 https://sustainabledevelopment.un.org/post2015/transformingourworld

[34] United Nations „Sustainable Development in an Ageing World: A call to UN Member States on the development agenda beyond 2015.“, 2015., preuzeto 20.01.2019 https://sustainabledevelopment.un.org/content/documents/6921Sustainable \%20development\%20in\%20an\%20ageing\%20world.pdf

[35] United Nations DP , Sustainable development that leaves no one behind“ preuzeto 05.01.2019, https://sustainabledevelopment.un.org/content/documents/2754713_July_P M_2._Leaving_no_one_behind_Summary_from_UN_Committee_for_Dev elopment_Policy.pdf

[36] European Central Bank, Economic Bulltin, pristupljeno 21.12.2018, https://www.ecb.europa.eu/pub/economic-bulletin/html/index.en.html

[37] A. Walker, A. Zaidi "New Evidence on Active Ageing in Europe", ZBW Leinbniz Inforamtion Centre for Economics, 2016, Intereconomics Volume 51, May/June 2016, Number 3 ·pp. 139-144 DOI: $10.1007 / \mathrm{s} 10272-016-0592-0$

[38] M. Naumanen, M. Tukiainen "Guided participation in ICT-education for seniors: Motivation and social support" 2009 39th IEEE Frontiers in Education Conference, San Antonio, TX, 2009, pp. 1-7.,doi: 10.1109/FIE.2009.5350544

[39] M.L. Fox „Let's give adults the benefits of digital skills“, pristupljeno 11.11.2017 https://www.theguardian.com/education/2012/jan/16/digitalliteracy-for-adults,

[40] D. Padilla-Góngora, R. López-Liria, et al. „Habits of the Elderly regarding Access to the New Information and Communication Technologies“, Procedia - Social and Behavioral Sciences, Volume 237, 21 February 2017, Pages 1412-1417, DOI: 10.1016/j.sbspro.2017.02.206

[41] E.Hernández-Encuentra, M. Pousada, B. Gómez-Zúñiga "ICT and Older People: Beyond Usability”, Educational Gerontology, 35:3, str. 226-245, 2009., DOI: 10.1080/03601270802466934

[42] A. González, M. Paz Ramírez, V. Viadel „Attitudes of the Elderly Toward Information and Communication Technologies“, Educational Gerontology No 38. str. 585-594, 2012. DOI 1080/03601277.2011.595314

[43] B. Xie "Information Technology Education for Older Adults as a Continuing Peer-Learning Process: A Chinese Case Study", Educational Gerontology, 33:5, 429-450, 2007, DOI: 10.1080/03601270701252872 
[44] J. Čurin „Specifičnosti poučavanja osoba treće životne dobi: Podsjetnik za nastavnike“, Andragoški glasnik, Vol.14., br. 1, 2010, str. 73-80

[45] L. Abad-Alcalá „Media Literacy for Older People facing the Digital Divide: The e-Inclusion Programmes Design“ Comunicar, 2014 Jan 1;21(42):173-180.

[46] P. Silva, AD. Matos, R. Martinez-Pecino „E-Inclusion: Beyond individual sociodemographic characteristics“, 2017. PLoS ONE 12(9): e0184545.https://doi.org/10.1371/journal. pone.0184545

[47] R.S. Sharma, et al."Digital literacy and knowledge societies: A grounded theory investigation of sustainable development", Telecommunications Policy, Volume 40, Issue 7, July 2016, Pages 628-643, doi 10.1016/j.telpol.2016.05.003

[48] R.S. Sharma, L.G. Malone, C. Guan and A. Dattakumar "A Maturity Model for Digital Literacies and Sustainable Development, Advanced Methodologies and Technologies in Library Science", Information Management, and Scholarly Inquiry, Copyright: (C) 2019 |Pages: 13, DOI: 10.4018/978-1-5225-7659-4.ch006

[49] L. Rathman „Evaluation of ICT skills \& Elderly People's Motivation in SATKA-project"“, svibanj, 2013, Otaniemi, preuzeto 5.12.2017, http://www.theseus.fi/bitstream/handle/10024/63420/Rahman_Lutfor.pdf;s equence $=1$

[50] S. Grom „Digital Skills for the Digital Economy“, Department of Culture, Media \&Sport, UK, 2016., preuzeto 10.10.2018, http://www.coe.gouv.fr/IMG/pdf/COE-UK_Digital_Skills_-S-_Grom.pdf

[51] Center for Economics and Business Research „The economic impact of Digital Inclusion in the UK, A report for Good Things Foundation", September 2018 preuzeto 10.05.2019.

https://www.goodthingsfoundation.org/sites/default/files/researchpublications/the_economic_impact_of_digital_inclusion_in_the_uk_final submission_stc_0.pdf

[52] State of Czech Republic „A new strategy for promoting digital literacy“, http://www.cedefop.europa.eu/en/news-and-press/news/czech-republicnew-strategy-promoting-digital-literacy, preuzeto 19.11.2018.

[53] R. Petrauskas, A. Kiskiene, T. Bileviciene "e-Inclusion IN LITHUANIA: STATE POLICY APPROACH", https://zaguan.unizar.es/record/4286/files/BOOK--2009-009.pdf\#page=73

[54] R. Petrauskas, T. Bilevičienè, A. Kiškiene "E-Inclusion as the part of egovernment evelopment in Lithuania", VIEŠOJI POLITIKA IR ADMINISTRAVIMAS, 2008. Nr. 23 
[55] IBSA (Innovation and Business Skills Australia) "Digital literacy and eskills: participation in the digital economy", preuzeto 4.1.2019. https://www.researchgate.net/publication/275522942_Digital_Literacy_an d_E-Skills_Participation_in_the_Digital_Economy

[56] State of Mexico „Digital Inclusion and Literacy Pilot Program“, 2014. www.oecd.org/gov/mexico-ICT-policy.pdf; www.oecd.org/gov/mexicointerministerial-commission-egov.pdf; www.oecd.org/gov/mexicodigital-strategy.pdf; http://www.oecd.org/gov/mexico-digital-literacy.pdf, preuzeto 19.11.2018.

[57] Actua, ,Why Canada needs a national digital literacy strategy“, November 2015, 7th Canadian Science Policy Conference, preuzeto 20.11.2018, https://www.sciencepolicy.ca/sites/default/files/digital_literacy__why_canada_needs_a_national_digital_literacy_strategy.pdf

[58] S. Reader „Digital Inclusion and Digital Literacy in the United States: A Portrait from PIAAC's Survey of Adult Skills“, 2015, preuzeto 29.11.2018. https://static1.squarespace.com/static/51bb74b8e4b0139570ddf020/t/551c3 e82e4b0d2fede6481f9/1427914370277/Reder_PIAAC.pdf

[59] European Commission „Boosting the economic and social e-Inclusion of the growing over 65 group", 2015., preuzeto 05.01.2019 https://ec.europa.eu/regional_policy/en/projects/belgium/boosting-theeconomic-and-social-e-Inclusion-of-the-growing-over-65-group

[60] R. Evangelista, P. Guerrieri, V. Meliciani "The economic impact of digital technologies in Europe", Italy Economics of Innovation and New Technology, Volume 23, 2014 - Issue 8, Pages 802-824, DOI: $10.1080 / 10438599.2014 .918438$

[61] Y. Eshet „Digital Literacy: A Conceptual Framework for Survival Skills in the Digital era", Jl. of Educational Multimedia and Hypermedia (2004) 13(1),93-106

[62] G. Naegele, E. Schnabel, et al. "Measures for social inclusion of the elederly: The case of volunteering", Eurofund, veljača 2011, preuzeto 3.3.2018. sa https://www.eurofound.europa.eu/publications/report/2010/quality-of-lifesocial-policies/measures-for-social-inclusion-of-the-elderly-the-case-ofvolunteering-working-paper

[63] A. Siren, S. Grønborg Knudsen "Older Adults and Emerging Digital Service Delivery: A Mixed Methods Study on Information and Communications Technology Use, Skills, and Attitudes", Journal of Aging \& Social Policy, Volume 29, 2017 - Issue 1, pages 35-50, doi: $10.1080 / 08959420.2016 .1187036$ 
[64] M. Negreiro „Bridging the digital divide in the EU“, European Parliamentary Research Service, Member's Research Service, Briefing, December 2015, European Parliament, preuzeto 1.10.2018. http://www.europarl.europa.eu/RegData/etudes/BRIE/2015/573884/EPRS BRI(2015)573884_EN.pdf

[65] N. Žajdela Hrustek "Multidimensional and multiperspective approach for monitoring e-inclusion", Doctoral thesis, Faculty of Organization and Informatics, Varaždin, 2015 